\title{
Photonic Crystals and Microdisk Cavities Based on GaInAsP-InP System
}

\author{
Toshihiko Baba, Member, IEEE
}

(Invited Paper)

\begin{abstract}
This paper presents a preliminary guide to realize microcavity semiconductor lasers exhibiting spontaneous emission control effects. It includes: 1) theoretical consideration on the effects; 2) processing techniques for semiconductor microcavities; and 3) some demonstrations of photonic crystal and microdisk cavity. It was shown that, even with a spectral broadening of electron transition, thresholdless lasing operation and alternation of spontaneous emission rate are expected in a cavity satisfying the single mode condition that only one mode is allowed in the transition spectrum. An ideal three-dimensional (3-D) photonic crystal has the potentiality for realizing this condition. In twodimensional (2-D) crystals and microdisk cavities, thresholdless operation is also expected, but the alternation of spontaneous emission rate may be negligible due to the insufficient optical confinement. In the experiment, some processing techniques for GaInAsP-InP system were investigated and methane-based reactive ion beam etching was selected because of the smooth sidewalls and adaptability to arbitrary structures. A GaInAsP-InP 2-D photonic crystal constructed by submicron columns was fabricated using this method. Owing to the slow surface recombination of this material, a polarized photoluminescence and peculiar transmission spectra were observed at room temperature (RT), which can be explained by a photonic band calculation. However, some technical improvement is necessary for clear demonstration of photonic bandgap, which is minimally required for device applications. In contrast to this, a GaInAsP-InP microdisk cavity of $2 \mu \mathrm{m}$ in diameter, which corresponds to the cavity volume 2.5 times the single-mode condition, has achieved RT lasing with threshold current as low as $0.2 \mathrm{~mA}$. Further reduction of dameter and realization of continuous-wave $(\mathrm{CW})$ operation will provide a significant regime for the observation of spontaneous emission control effects.
\end{abstract}

Index Terms-GaInAsP-InP, microcavity, microdisk, photonic crystals, spontaneous emission control, semiconductor laser.

\section{INTRODUCTION}

$\mathbf{S}$ EMICONDUCTOR microcavities have been attracting attention in these ten years because of the following reasons: 1) possibility of ultra-small lasers with low power consumption suitable for large scale integration; 2) potentiality of thresholdless lasing operation owing to the high efficiency coupling of spontaneous emission into one lasing mode; and 3) physical interest for the strong coupling of electron and photon states resulting in the alternation of spontaneous emission rate

Manuscript received April 25, 1997. This work was supported in part by the Support Center for Advanced Telecommunications, in part by Ozawa and Yoshikawa Memorial Electronics Research Foundation, and in part by Nippon Sheet Glass Foundation for Material Science and Engineering.

The author is with the Yokohama National University, Division of Electrical and Computer Engineering, Hodogayaku, Yokohama 240, Japan.

Publisher Item Identifier S 1077-260X(97)07198-0. and the quantum reversible process of spontaneous emission, i.e., Rabi oscillation. As for the first reason, much effort has been made toward the development of vertical-cavity surface-emitting lasers (VCSEL's) for light sources in optical interconnect and fiber communication systems. This paper focuses on microcavities for the latter two reasons, which have been discussed as research issues of "spontaneous emission control."

The history of this concept has started from the suggestion by Purcell [1] and progressed through quantum theoretical consideration for electron and photon interaction [2] and experiments using metal cavities and micrometer/millimeter waves [3]-[5]. After 1980, some authors have moved their targets to lightwave frequencies, and reported a consideration on closed cavity light emitters [6], proposal of photonic crystals [7]-[9], and some observations of spontaneous emission control in dielectric planar microcavities with dye solution [10], [11]. Just after the success of VCSEL's [12]-[14], which have opened the possibility of semiconductor microcavities, some effects of spontaneous emission control in a VCSEL-type planar microcavity have been clearly demonstrated using a narrow transition spectrum from excitons in quantum-wells at low temperature [15], [16]. The intensity and radiation pattern of spontaneous emission were strongly modulated using on and off resonant conditions of the cavity. Various theoretical and experimental studies have followed the demonstration, i.e., rate equation analyzes of general characteristics of microcavity lasers [16]-[20], quantum treatment for modes in microcavities [21], estimation of spontaneous emission factor and spontaneous emission rate in air-post VCSEL's [22], [23], discussion on photon recycling effect [24]-[26], observations of spontaneous emission control at room temperature [27], [28] and Rabi splitting [29] in VCSEL-type planar microcavities, photo-pumped lasing in a submicron-diameter air-post VCSEL [30], low threshold electrically-pumped lasing in microdisk lasers [31], [32], and trial fabrications of semiconductor photonic crystal for lightwave frequencies [33]-[36]. Thus, the concept has become one of the major topics in both physics and optoelectronics [37]. In spite of this situation, however, its effectiveness in actual lasers is still not clear. First of all, it is due to the following essential problem. Most of the peculiar effects of spontaneous emission control are predicted by presupposing single electron and single photon interaction. In actual, there exists a large spectral broadening in bandto-band transition of electrons in semiconductors at room temperature. Likewise, many photon states, namely, many electromagnetic modes are allowed in structurally imperfect 
cavities with weak optical confinement. Careful consideration for these complications is necessary for quantitative discussion of expected effects.

There also exist problems for semiconductor materials and fabrication techniques of cavity structures. Typical materials so far employed for microcavities are $\mathrm{Ga}(\mathrm{In}) \mathrm{As}-\mathrm{AlGaAs}$ and GaInAsP-InP systems, which have been widely studied for various applications of optical technologies. Advantages of $\mathrm{Ga}(\mathrm{In}) \mathrm{As}-\mathrm{AlGaAs}$ are relatively easy realization of lasing operation in any cavities owing to the large optical gain, easy fabrication of fine structures by fully established dry etching techniques such as chlorine-based reactive ion beam etching (RIBE) [38], chemically assisted ion beam etching (CAIBE) [39], etc., and easy evaluation of the effects by highly sensitive detectors and imaging equipments available for wavelength shorter than $1 \mu \mathrm{m}$. However, this material system has a problem of large surface recombination, which seriously degrades the internal quantum efficiency of microcavity lasers having relatively large surface area for their volume [30]. Nevertheless, a large refractive index difference $\Delta n$ (or index ratio) between semiconductor and air (or another low index material) is crucial to realize strong optical confinement with high quality factor $Q$ in microcavities. For this reason, the research described in this paper has concentrated on microcavities based on GaInAsP-InP system. It is known that surface recombination of this system is one to two orders of magnitude slower than that of $\mathrm{Ga}(\mathrm{In}) \mathrm{As}-\mathrm{AlGaAs}$. However, there have been a limited number of reports on microcavity fabrication of this system. In contrast to that for $\mathrm{Ga}(\mathrm{In}) \mathrm{As}-\mathrm{AlGaAs}$, fine dry etching techniques for this system are still under developing.

The aim of this paper is to show a preliminary guide to realize semiconductor microcavity lasers exhibiting spontaneous emission control effects beneficial to their performance. In Section II, some simple expressions for the effects are derived with an ideal cavity model, which indicate that the thresholdless lasing operation and alternation of spontaneous emission rate are possible even with a spectral broadening of electron transition, if the cavity is so small as to satisfy the single mode condition that only one cavity mode overlaps with the transition spectrum. Such a small cavity is equivalently realized as the volume of a strongly confined mode field in actual microcavities. Microcavity structures are roughly categorized into photonic crystals and whispering gallery mode cavities. Both of them have three-dimensional (3-D) complicated shapes and need some anisotropic etching or selforganization techniques for their fabrications. In Section III, characteristics of four etching techniques are described and their capabilities for microcavity fabrication are discussed. In Section IV, the possibility of spontaneous emission control in photonic crystals is discussed with the simple treatment of modes, and it will be concluded that almost single-mode condition can be realized in higher dimensional crystals with a wide photonic bandgap. Design and fabrication were performed for two-dimensional (2-D) crystals constructed by submicron columns to obtain some elementary results, although they are still too difficult technically to apply to lasers. A photonic bandgap of the columns was observed by an equivalent transmission measurement. In Section V, those for whispering gallery mode cavities are described. The discussion will be concentrated on microdisk cavities, since this type easily achieves electrically pumped lasing operation with present technologies. Ultralow-threshold lasing characteristics in very small devices fabricated in this study will be presented. The status of this result for spontaneous emission control and ultimate performance are discussed.

\section{SPONTANEOUS EMISSION CONTROL IN IDEAL MiCROCAVITY}

Let me consider a semiconductor active region put in a cavity surrounded by perfectly conductive walls. When the cavity is large enough, many modes distribute continuously over the frequency space. The net stimulated emission rate $R_{\mathrm{st}}$ for a laser mode and average spontaneous emission rate $R_{\mathrm{sp}}$ for all modes are given by (see Appendix A)

$$
\begin{aligned}
& R_{\mathrm{st}}=\Gamma S \frac{\pi c^{2} e^{2} \mu_{0}^{2} \omega_{l} F_{\mathrm{st}}}{3 n_{a}^{2} \varepsilon_{0}} \\
& R_{\mathrm{sp}}=\frac{e^{2} \mu_{0}^{2} \omega_{s}^{3} n_{\mathrm{eff}}}{3 \pi c^{3} \varepsilon_{0}}
\end{aligned}
$$

where $\Gamma$ is the optical confinement factor into active region, $S$ the photon density of laser mode, $\mu_{0}$ the average amplitude of dipole moment, $\omega_{l}$ and $\omega_{s}$ the resonant frequency of laser mode and the center frequency of electron transition spectrum, respectively, $F_{\mathrm{st}}$ the gain spectrum, $n_{a}$ the refractive index of active region, $n_{\mathrm{eff}}$ the average effective index of modes and $c$ the vacuum velocity of light. Spontaneous emission rate can be changed by the effective index $n_{\text {eff }}$ [40].

Next, let me consider a microcavity with volume $V_{c}$, in which cavity modes distribute discretely. In such a cavity, (1) is still valid, while (2) is changed. If the electron transition spectrum is approximated by Lorentzian function with fullwidth at half-maximum (FWHM) of $\Delta \omega_{s}$, semi-single mode condition that only one mode with degenerate two polarizations is allowed inside the spectrum is expressed as

$$
\left[\frac{\lambda_{s}}{2 n_{\mathrm{eq}}}\right]^{3} \leq V_{c} \leq \frac{\lambda_{s}^{4}}{2 \pi^{2} n_{\mathrm{eq}}^{3} \Delta \lambda_{s}}
$$

where $n_{\mathrm{eq}}$ is the equivalent index of the mode, $\lambda_{s}=2 \pi c / \omega_{s}$ and $\Delta \lambda_{s} / \lambda_{s}=\Delta \omega_{s} / \omega_{s}$. The left side of (3) is the cutoff condition of cavity. $R_{\mathrm{sp}}$ in such a cavity is then (see Appendix A)

$$
R_{\mathrm{sp}}=\frac{2 \pi e^{2} \mu_{0}^{2} \omega_{l} \Gamma_{r}}{3 \varepsilon_{0} n_{\mathrm{eq}}^{2} V_{c}} \frac{\Delta \omega_{s} / 2}{\pi\left[\left(\omega_{s}-\omega_{l}\right)^{2}+\left(\Delta \omega_{s} / 2\right)^{2}\right]}
$$

where $\Gamma_{r}$ is the relative confinement factor. Now, $\gamma$ is defined as the ratio of spontaneous emission rate in a cavity to that in a bulk semiconductor with index of $n_{\text {ref. }}$. By comparing (4) with (2),

$$
\begin{aligned}
\gamma & =\frac{n_{\mathrm{eq}}}{n_{\mathrm{ref}}} \cdot \frac{4 \pi \Gamma_{r} c^{3}}{n_{\mathrm{eq}}^{3} V_{c} \omega_{s}^{2} \Delta \omega_{s}} \\
& =\frac{n_{\mathrm{eq}}}{n_{\mathrm{ref}}} \cdot \frac{\Gamma_{r} \lambda_{s}^{4}}{2 \pi^{2} n_{\mathrm{eq}}^{3} V_{c} \Delta \lambda_{s}}
\end{aligned}
$$

where $\omega_{l}=\omega_{s}$ was assumed. If the mode suffers losses, it should be characterized by a normalized spectrum function with broadening $\Delta \omega_{l}$. Even under such condition, however, 
(5) is still valid when $\Delta \omega_{l} \ll \Delta \omega_{s}$. For $\Delta \omega_{l} \gg \Delta \omega_{s}, \Delta \omega_{s}$ in (5) is replaced by $\Delta \omega_{l}$ and

$$
\gamma=\frac{n_{\mathrm{eq}}}{n_{\mathrm{ref}}} \cdot \frac{4 \pi \Gamma_{r} c^{3}}{n_{\mathrm{eq}}^{3} V_{c} \omega_{s}^{2} \Delta \omega_{l}}=\frac{n_{\mathrm{eq}}}{n_{\mathrm{ref}}} \cdot \frac{\Gamma_{r} \lambda_{s}^{3} Q}{2 \pi^{2} n_{\mathrm{eq}}^{3} V_{c}}
$$

where $Q \equiv \omega_{l} / \Delta \omega_{l}$. It is seen from (5) and (6) that $\gamma$ is dominated by a wider spectrum. Equation (5) is used for transition spectra of semiconductor at room temperature, which is much broader than resonant spectra of a high $Q$ microcavity. On the other hand, (6) should be used for narrow transition spectra from excitons at low temperature. If the degenerate polarizations are split in an asymmetric cavity and only resonant frequency for one polarization is tuned to the transition spectrum, the right side of (4)-(6) is decreased by factor $1 / 2$.

Spontaneous emission factor $C$ [22], [23], [41] is defined as the ratio of spontaneous emission rate for one mode to that for all the modes. From (2) and (4),

$$
C=\frac{4 \pi \Gamma_{r} c^{3}}{n_{\mathrm{eq}}^{3} V_{c} \omega_{s}^{2} \Delta \omega_{s}}=\frac{\Gamma_{r} \lambda_{s}^{4}}{2 \pi^{2} n_{\mathrm{eq}}^{3} V_{c} \Delta \lambda_{s}} .
$$

Factor $1 / 2$ is also necessary for this expression when a singlepolarized mode is considered. This means that the upper limit of $C$ factor is $1 / 2$ for a single-polarized mode. To raise this value to 1 , an asymmetric cavity and/or anisotropic orientation of dipoles must be used [23]. Equation (7) has the same form as that of (5) except for factor $n_{\mathrm{eq}} / n_{\text {ref. }}$. Ignoring this factor, changes of $\gamma$ and $C$ for a mode with the polarization degeneracy are calculated with normalized cavity volume $\tilde{V}$, as shown in Fig. 1. When $\tilde{V}$ is larger than 1, multiple cavity modes are allowed in the spectrum, which cancel the increase of $V_{c}$ in (5) and bring $\gamma$ close to 1 . Product $\gamma C$ is inversely proportional to $\tilde{V}$. Let me consider a GaInAsP-InP laser with $\lambda_{s}=1.55 \mu \mathrm{m}, \Delta \lambda_{s}=80 \mathrm{~nm}, n_{\mathrm{eq}}=n_{\mathrm{ref}}=3$ and $\Gamma_{r}=2$, as an example. Then, $C=1$ is obtained for $V_{c}=0.51^{3} \mu \mathrm{m}^{3}$ and $\gamma=20$ for cutoff condition $V_{c}=0.26^{3} \mu \mathrm{m}^{3}$.

Lasing characteristics are obtained by solving the almost standard rate equations

$$
\begin{aligned}
& \frac{d N}{d t}=-\Gamma S G(N)-\left(\frac{A_{a}}{V_{a}} v_{s} N+\gamma B N^{2}\right)+\frac{I}{e V_{a}} \\
& \frac{d S}{d t}=\Gamma S G(N)+\gamma C B N^{2}-\frac{S}{\tau_{p}}
\end{aligned}
$$

where $N$ is the carrier density in the active region, $v_{S}$ the surface recombination velocity, $A_{a}$ the surface area of active region, $I$ the injection current and $\tau_{p}$ the photon lifetime. Equation (1) for stimulated emission rate was rewritten as $\Gamma S G(N)$, where $G(N)$ is the gain coefficient. Equation (4) for spontaneous emission rate was approximated by $\gamma B N^{2}$, where $B$ is the radiative recombination coefficient. Only the surface recombination was taken into account as a nonradiative recombination process, considering a relatively low carrier density in a high- $Q$ cavity. Fig. 2(a) shows photon density $S$ versus pump density $I / e V_{a}$. Parameters used for calculation and meaning of notations A-F are summarized in Table I. The thresholdless lasing characteristic [6], [11], [16], [17] is observed for $C=1$ when neglecting surface recombination (curves D-F). Fig. 2(b) shows corresponding extinction power of laser mode $P \equiv \hbar \omega_{l} S V_{a} / \tau_{p}$ versus current $I$. Since

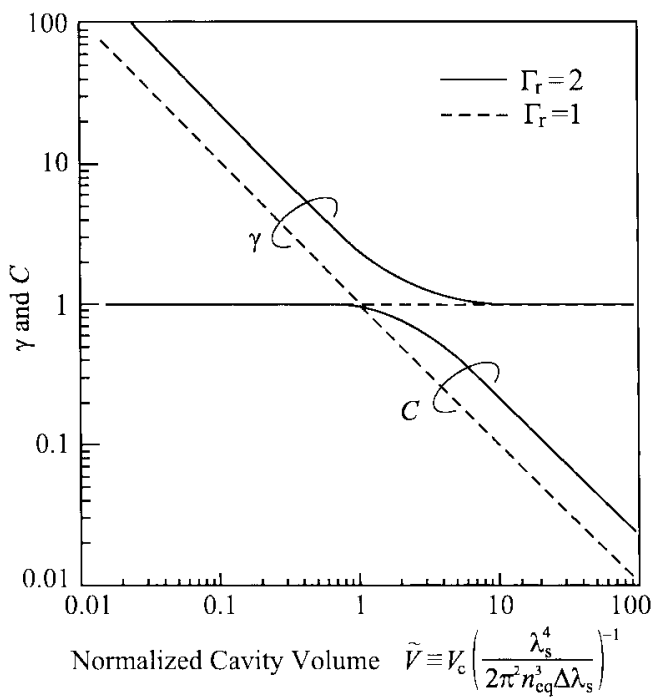

Fig. 1. Alternation ratio of spontaneous emission rate $\gamma$ and spontaneous emission factor $C$ calculated with normalized cavity volume $\tilde{V}$.

threshold current $I_{\text {th }}$ is given by $e \gamma B N_{\text {th }}^{2} V_{a}$, it is simply reduced by decreasing $V_{a}$ simultaneously with $V_{c}$. Once the cavity satisfies $\tilde{V}<1$, decrease of $V_{c}$ and increase of $\gamma$ cancel with each other so that $I_{\mathrm{th}}$ is no more reduced. When a large surface recombination is considered, efficiency at low pump density and at low injection current is seriously degraded. Suppression of surface recombination is crucial for the thresholdless operation. In Fig. 2, $\gamma>1$ is assumed for smaller cavities. However, $\gamma \ll 1$ may also be possible when $\omega_{l}$ is detuned from $\omega_{s}$ and/or distribution of modes is strongly modulated so that a limited number of modes overlap with the transition spectrum. For such condition, $I_{\mathrm{th}}$ is simply reduced independenly of $V_{c}$.

High-output power from microcavity lasers is often doubted because of the small volume. In essential, however, they can achieve comparably high power for the small volume. Electrical resistance of lasers is inversely proportional to the dimension, and thermal resistance and ohmic contact resistance the square of dimension, while injection current is proportional to the cubic of dimension. This results in a small temperature increase for a certain pump density or allows a very high pump density and photon density in a microcavity.

Photon lifetime $\tau_{p}$ is one of the important parameters that dominate the response of lasers. For microcavity lasers, both increase of $\tau_{p}$ by "a high- $Q$ " and decrease by "a short cavity" can be supposed. Especially, extreme increase of $\tau_{p}$ is an interest regime that gives rise to the Rabi oscillation. [29] However, it also causes a narrow modulation bandwidth and low external efficiency. Extreme decrease of $\tau_{p}$ causes a high threshold current. Since this discussion may conclude the design choice, the special change of $\tau_{p}$ is not considered here. Fig. 3 shows modulation characteristics calculated from (8) and (9). When $C$ is equal to 1 , it exhibits a mixed characteristic of light-emitting diodes (LED's) and laser diodes. For $\gamma=$ 1 , spontaneous emission lifetime $\tau_{s} \equiv 1 / R_{\mathrm{sp}}$ limits the modulation bandwidth below threshold (A-D). For $\gamma>1, I_{\text {th }}$ is clamped against further reduction of $V_{c}$ and the enhanced spontaneous emission rate allows LED's to have response comparable to those of laser diodes ( $\mathrm{E}$ and $\mathrm{F}$ ). For example, 3-dB bandwidth exceeds $7 \mathrm{GHz}$ for $\gamma=10$ and $I=0.1$ 


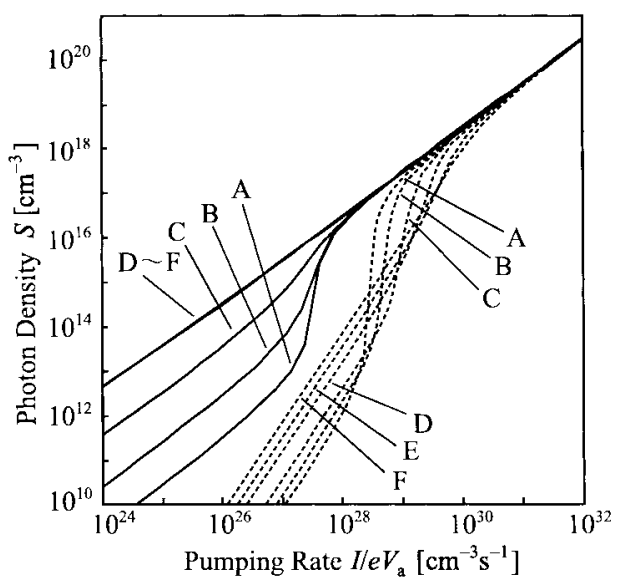

(a)

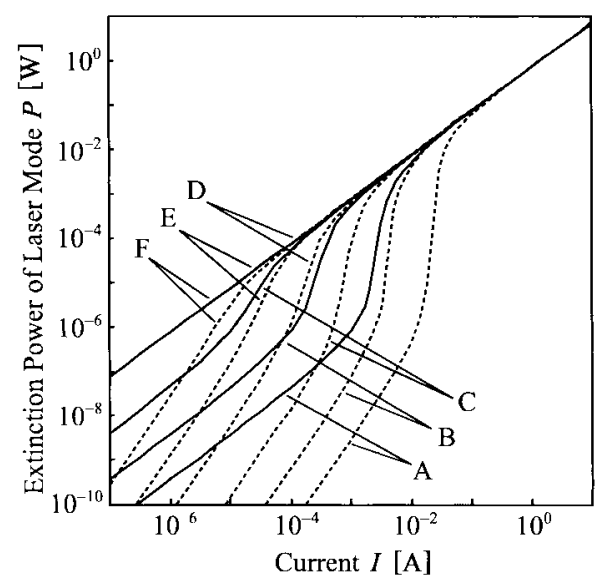

(b)

Fig. 2. Lasing characteristics of microcavity laser. For solid and dashed curves, $v_{s}=0$ and $2 \times 10^{5} \mathrm{~cm} / \mathrm{s}$ are assumed, respectively.

$I_{\mathrm{th}}(\mathrm{E})$. The stimulated emission dominates the characteristic and the relaxation oscillation limits the bandwidth for $I>I_{\mathrm{th}}$. However, the relaxation oscillation itself is suppressed when $C=1$ (D and E) [17]. It is caused by the simultaneous increase or decrease of carrier and photon densities. Since the ratio of spontaneous emission to stimulated emission is still high just above threshold, the modulated wave form is influenced by the lifetime $\tau_{s}$. For higher injection level, the bandwidth is simply increased by the increase of photon density. If the current can be over $100 I_{\text {thl }}$, the bandwidth is enhanced to $100 \mathrm{GHz}$.

Some results shown above are different from those reported previously [16], [18]. This is because the change of $C$ factor independently of cavity volume, which was sometimes assumed in those papers, seems to be difficult, if one accepts the broadening of transition spectrum of semiconductor.

Finally, let me discuss the photon recycling in microcavities. It is the effect that spontaneous emission confined in a cavity is reabsorbed and recycled as carriers [42]. Under a strong coupling regime of electron and photon states in a cavity with $C=1$ and ultrahigh- $Q$, this effect gives rise to the Rabi oscillation. Under a weak coupling regime in a realistic cavity obtained at present, this effect is expected to serve for the economy of injection current and the threshold reduction in laser diodes [24], [25]. However, such recycling cannot be considered in a cavity with $C=1$, because there remains no
TABLE I

\begin{tabular}{|c|c|c|c|c|}
\hline \multicolumn{2}{|c|}{$\begin{array}{l}\lambda_{\mathrm{s}}=1.55 \mu \mathrm{m} \\
\Delta \lambda_{\mathrm{s}}=80 \mathrm{~nm} \\
n_{\mathrm{eq}}=3\end{array}$} & $\begin{array}{l}\Gamma=0.04 \\
\Gamma_{\mathrm{r}}=2 \\
\tau_{\mathrm{p}}=3 \mathrm{ps}\end{array}$ & \multicolumn{2}{|c|}{$\begin{array}{l}G=5 \times 10^{-6} \mathrm{~cm}^{3} / \mathrm{s} \\
B=2.5 \times 10^{-10} \mathrm{~cm}^{3} / \mathrm{s} \\
N_{0}=2 \times 10^{18} \mathrm{~cm}^{-3}\end{array}$} \\
\hline A & $V_{\mathrm{c}}=270 \mu \mathrm{m}^{3}$ & $S_{\mathrm{a}}=3.34 \mu \mathrm{m}^{2}$ & $C=0.001$ & $\gamma=1$ \\
\hline B & 27 & 0.72 & 0.01 & 1 \\
\hline C & 2.7 & 0.151 & 0.1 & 1 \\
\hline D & 0.27 & 0.0334 & 1 & 2 \\
\hline E & 0.027 & 0.0072 & 1 & 20 \\
\hline $\mathrm{F}$ & 0.0027 & 0.00151 & 1 & 200 \\
\hline
\end{tabular}

spontaneous emission of nonlasing modes, which contributes to the recycling. The recycling will be efficient in a relatively large cavity having many nonlasing modes with loss slightly larger than that for the lasing mode. The recycling fades out at higher injection level at which gain spectrum overlaps with spontaneous emission spectrum. Therefore, the threshold reduction is limited to nearly $1 / 2$ of that without recycling. For lower injection level, extreme high external efficiency in LED's is expected by the large absorption of spontaneous emission [26].

\section{Processing of Microcavities}

In actual microcavities, volume smaller than $1 \mu \mathrm{m}^{3}$ is equivalently realized as the mode volume strongly confined around the active region using high reflectivity mirrors. The mirror reflectivity $R$ corresponds to the resonant spectral width $\Delta \omega_{l}$, and dominates the upper limit of the effects under the critical condition $\Delta \omega_{l} \ll \Delta \omega_{s}$. Let me consider a Fabry-Perot cavity laser with mirror interval of $L$ and internal absorption loss of $10 \mathrm{~cm}^{-1}$. To suppress the mirror loss $\ln (1 / R) / L$ to comparable to the absorption loss, $R$ must be as high as 0.999 and 0.9999 for $L=1$ and $0.1 \mu \mathrm{m}$, respectively. However, to satisfy the critical condition, $R$ must be at least much higher than 0.534 and 0.939 for $L=1$ and $0.1 \mu \mathrm{m}$, respectively, when assuming $\lambda_{s}=1.55 \mu \mathrm{m}$ and $\Delta \lambda_{s}=80 \mathrm{~nm}$.

From the of viewpoint of mirror reflection, microcavity structures are categolized into two types; one confines the mode field by multiple reflection, while the other by total internal reflection. Vertical-cavity surface-emitting lasers (VCSEL's) and photonic crystals are the former type, while whispering gallery mode cavities are the latter type. So far, reported structures and fabrication techniques of microcavities based on GaInAsP-InP and some other materials are summarized in Table II. GaInAsP-InP VCSEL's have been approaching the practical device by the improvement of dielectric and/or semiconductor multilayer mirrors with reflectivity over 0.99 [43]-[47]. However, since most of them are employing planar or low mesa cavities having relatively weak optical confinement, they are not suitable for spontaneous emission control. To achieve the small-mode volume, complicated structures with large $\Delta n$ between semiconductor and air are often employed in photonic crystals and whispering gallery mode cavities. The surface recombination becomes serious in such structures with large surface area exposed to the air. For example, let me consider the internal quantum efficiency $\eta_{i}$ in a micro-column. From rate equations, $\eta_{i}$ is derived as

$$
\eta_{i}=1-\frac{A_{a} v_{s}\left(\sqrt{\left(A_{a} v_{s}\right)^{2}+4 V_{a} B(I / e)}-A_{a} v_{s}\right)}{2 V_{a} B(I / e)} .
$$




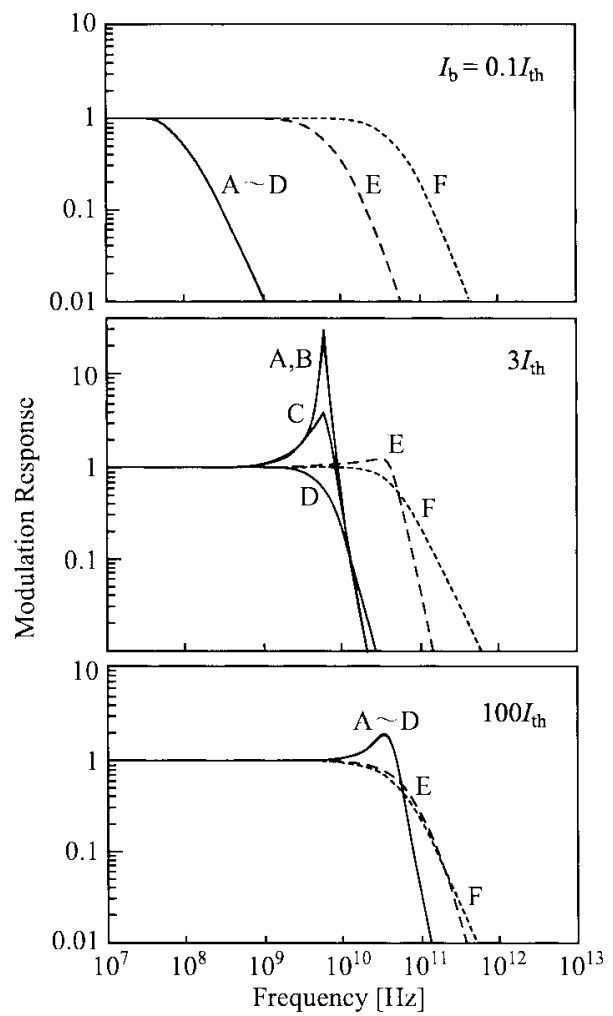

(a)

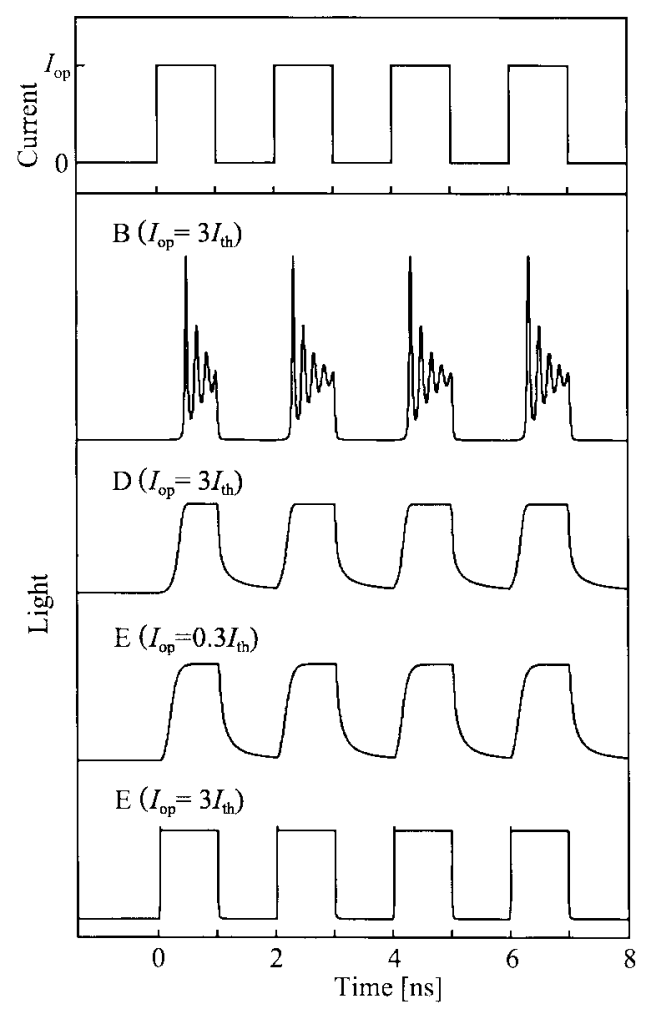

(b)

Fig. 3. Modulation characteristics of microcavity laser. Small-signal modulation with bias current of $I_{b}$ is assumed for (a), while zero-bias modulation for (b).

Fig. 4 shows $\eta_{i}$ with injection current $I$. It is seen that, if one desires a high efficiency for current lower than $1 \mathrm{~mA}, v_{s}<1$ $\times 10^{4} \mathrm{~cm} / \mathrm{s}$ is crucial. The velociry $v_{s}$ is strongly dependent on
TABLE II

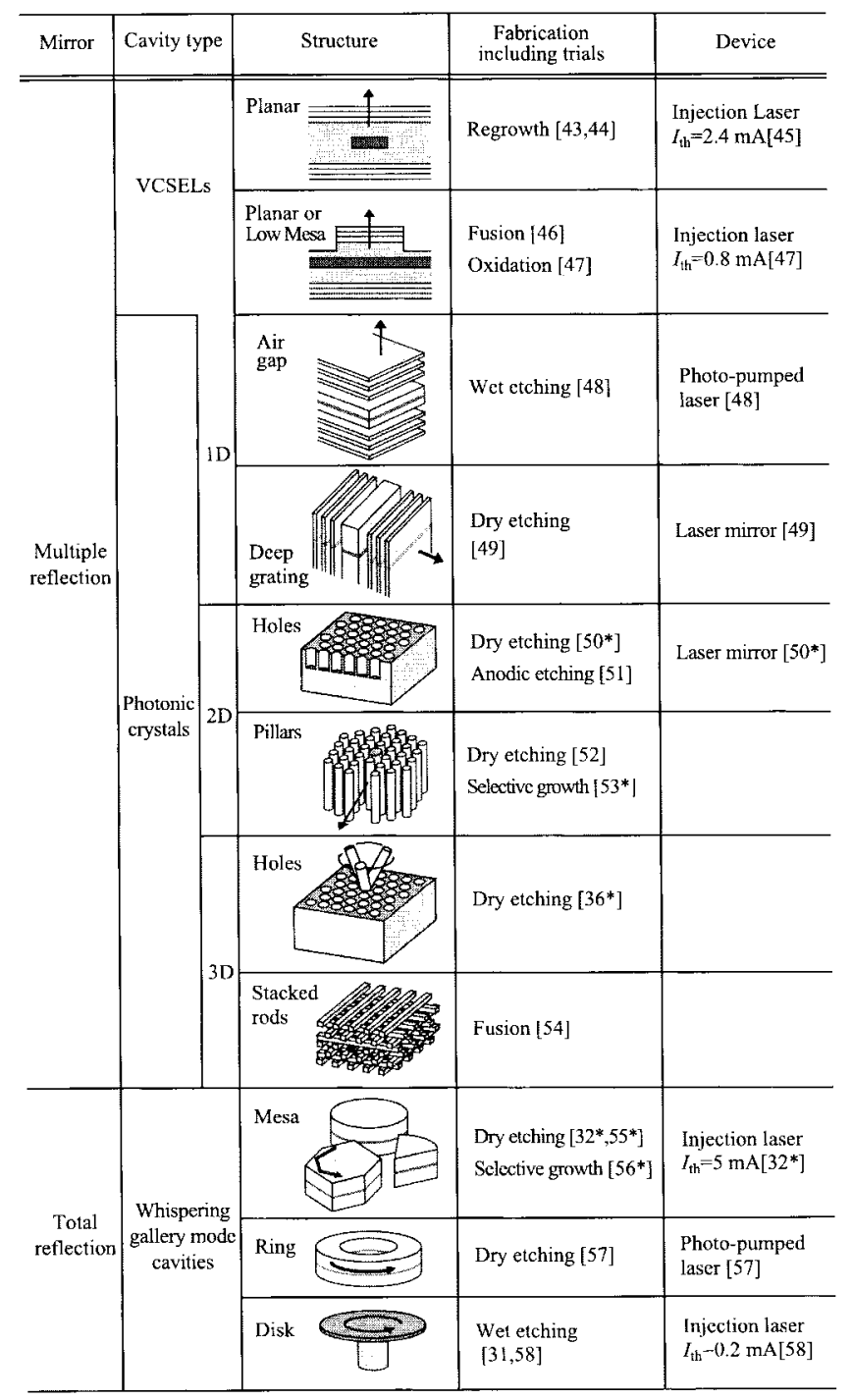

surface condition of semiconductor. Typical value for GaAsair interface is $1-5 \times 10^{5} \mathrm{~cm} / \mathrm{s}$, [59], [60] while that for InP-air interface $0.5-5 \times 10^{4} \mathrm{~cm} / \mathrm{s}$ [61]. Thus, a high efficiency seems to be difficult for $\mathrm{Ga}(\mathrm{In}) \mathrm{As}-\mathrm{AlGaAs}$, but can be expected for GaInAsP-InP.

In this paper, four typical anisotropic etching techniques for GaInAsP-InP have been investigated, i.e., $\mathrm{Cl}_{2}$-based RIBE [62]-[64], methane $\left(\mathrm{CH}_{4}\right)$-based RIBE [65], [66], selective wet chemical etching with hydrogen chrolide $(\mathrm{HCl})$ solution [67]-[69] and anodic chemical etching [51], [70]. For $\mathrm{Cl}_{2}$ RIBE, ultrahigh vaccum system ANELVA UHV-ECR [63], [64] was used. An advantage of this etching for GaInAsP-InP is the high etch rate and negligible retreat of mask edge. It allows aspect ratio over 20 with sidewalls faithful to the incident direction of ion beam. Fig. 5 shows a deep grating formed on $\mathrm{InP}$ substrate by this etching with microwave power of $300 \mathrm{~V}$, acceleration voltage of $300 \mathrm{~V}, \mathrm{Cl}_{2}$ gas pressure of $1.3 \times 10^{-4}$ torr and substrate temperature of 270 ${ }^{\circ} \mathrm{C}$. Electron beam (EB) resist Shipley SAL601 was used as a mask. Under this condition, etch rate for InP was as high as $500 \mathrm{~nm} / \mathrm{min}$. The grating has semiconductor linewidth of nearly $0.3 \mu \mathrm{m}$ and air space of $1.2 \mu \mathrm{m}$, which almost satisfy 


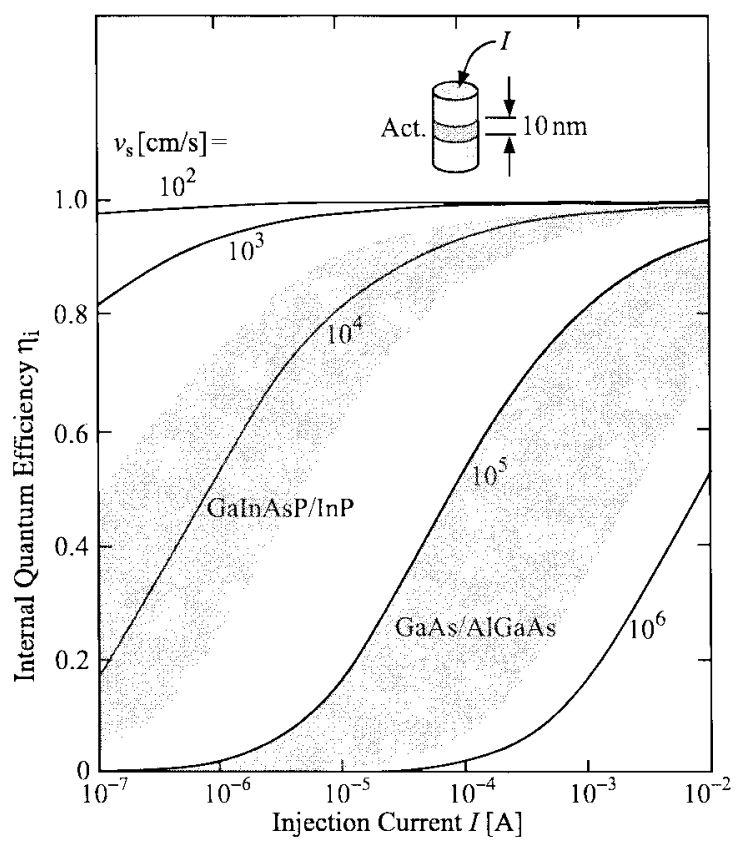

Fig. 4. Internal quantum efficiency of semiconductor micro-columns calculated with injection current.

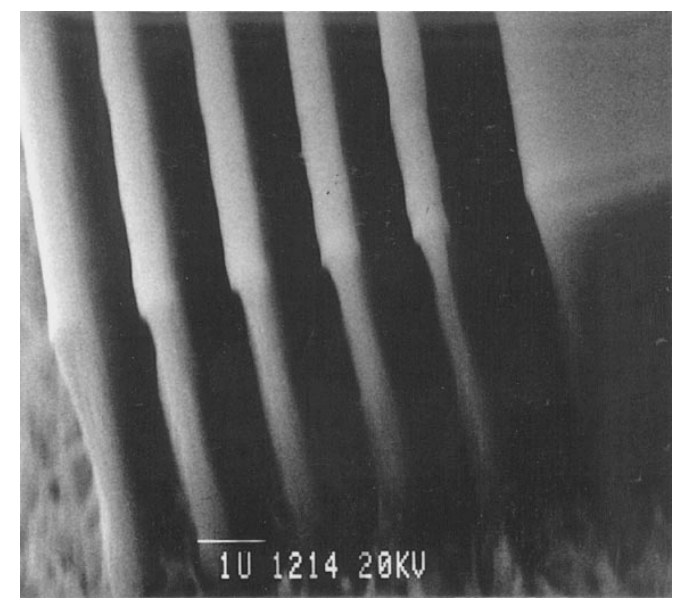

Fig. 5. Ultra-deep $3 \lambda_{s} / 4 n$ grating formed into InP substrate by $\mathrm{Cl}_{2}$ RIBE.

the three quater wavelength $\left(3 \lambda_{s} / 4 n\right)$ condition for $\lambda_{s}=$ $1.55 \mu \mathrm{m}$. Thus, the grating can be used as a multilayer mirror in a horizontal short cavity stripe laser [49]. To obtain $\lambda_{s} / 4 n$ condition, the space width must be $0.39 \mu \mathrm{m}$. For such narrow space, the etched depth was reduced to nearly $30 \%$ of that for broad area by the loading effect, as shown in Fig. 6. It was slightly improved by a higher acceleration voltage. The reflectivity of the $3 \lambda_{s} / 4 n$ grating was evaluated by measuring the threshold of stripe lasers with the grating for one facet and the cleaved end for another facet. Threshold current density was measured for various cavity lengths and compared with those of lasers with two cleaved facets, as shown in Fig. 7. From the fitting of theoretical curves, the effective reflectivity of the grating was evaluated to be $0.2-0.6$. The reflectivity of such mirror, calculated for plane waves using transfer matrix method [71], is over 0.999. One of the reason that degrades the effective reflectivity is the diffraction loss of reflected light in the air space; the reflectivity of $3 \lambda_{s} / 4 n$ grating cannot be higher than $0.75-0.85$ for guided waves in lasers [49].

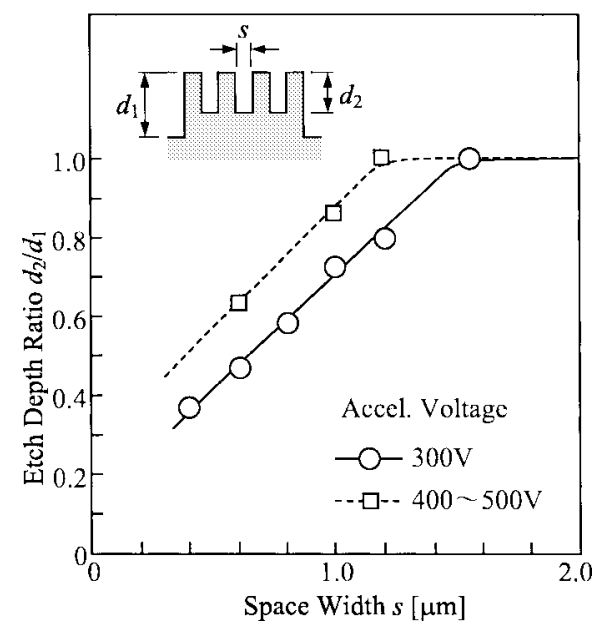

Fig. 6. Etch depth ratio measured for various space widths of deep grating formed by $\mathrm{Cl}_{2}$ RIBE. $d_{1}$ was deeper than $4 \mu \mathrm{m}$.

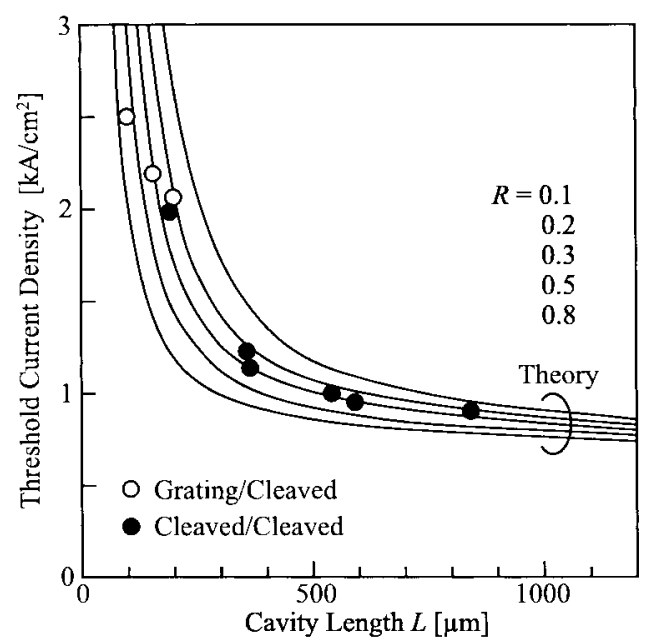

Fig. 7. Theoretical curves and experimental plots of threshold current density of stripe laser versus cavity length. The grating has five corrugations. A compressive-strained quantum-well wafer was used for experiment.

Another reason is the light scattering at etched sidewalls with roughness. Fig. 8(a) shows topographic image of a sidewall inside the grating obtained by a field-emission-type scanning electron microscope (SEM) [72]. The maximum amplitude of roughness $\sigma$ was $35 \mathrm{~nm}$. It is much larger than $20 \mathrm{~nm}$, which was observed for sidewalls of a large simple mesa formed by the same etching. It may be caused by a high vapor pressure of $\mathrm{InCl}_{2}$ and $\mathrm{InCl}_{3}$ inside the grating, which disturbs the smooth chemical reaction. Fig. 9 simulates reflection spectra of $3 \lambda / 4 n$ grating for plane waves. The unwanted light scattering at rough sidewalls was taken into account by the exponetial decay of reflection [73] and randomly generated phase error at each facet. The resonant behavior between stopbands becomes ambiguous even with $\sigma=5 \mathrm{~nm}$ and almost disappears with $\sigma \geq 20 \mathrm{~nm}$. The maximum reflectivity can be decreased to 0.7 with $\sigma=35 \mathrm{~nm}$. Although a lower acceleration voltage reduces the roughness [72], it also reduces the etch depth.

$\mathrm{CH}_{4}$-based RIBE is effective for smooth sidewalls. Compact etching apparatus Elionix EIS-200ER with mixture of $\mathrm{CH}_{4}$, $\mathrm{H}_{2}$, and Ar gaseous source and microwave power of 100 $\mathrm{W}$ was used. To reduce the deposition of carbon polymer at the etched surface and sidewalls, total gas pressure was 


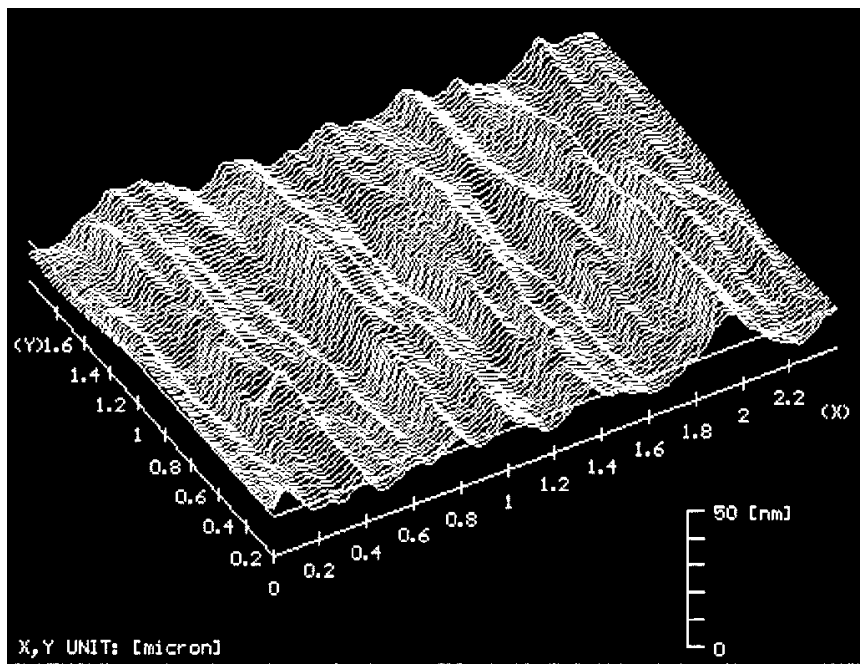

(a)

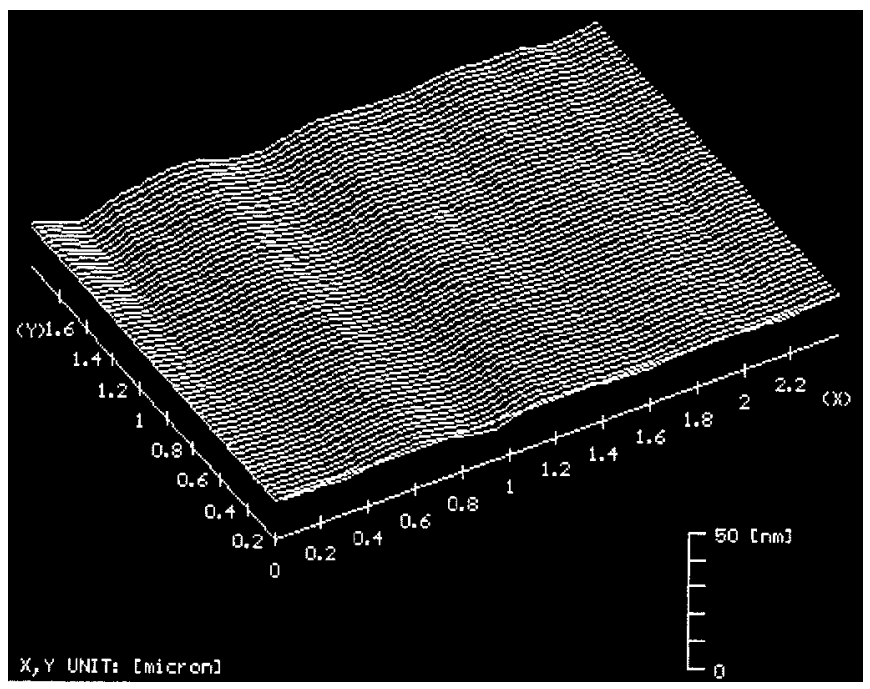

(b)

Fig. 8. Topographic image of sidewall inside deep grating etched by $\mathrm{Cl}_{2}$ RIBE (a) and that of sidewall of simple mesa etched by $\mathrm{CH}_{4}$-based RIBE (b).

suppressed to $7-10 \times 10^{-5}$ torr. Accordingly the gas flow rate was set to be small; rate of $\mathrm{CH}_{4}$ was varied from 0.5 to $0.75 \mathrm{sccm}$, while those of $\mathrm{H}_{2}$ and $\mathrm{Ar}$ were fixed to 0.9 and $0.3 \mathrm{sccm}$, respectively. The acceleration voltage was set to be $200 \mathrm{~V}$ or less to reduce the induced damage. Etch rate for InP takes the maximum value of $82 \mathrm{~nm} / \mathrm{min}$ with $\mathrm{CH}_{4}$ flow rate of $0.65 \mathrm{sccm}$. Etch rate for $1.3-\mu \mathrm{m}$-quaternary (Q) material was roughly estimated to be $30 \%$ of that for InP under the same etching condition. These values are also decreased by the loading effect similar to $\mathrm{Cl}_{2}$ RIBE. The damage depth of etched surface was evaluated from the photoluminescence (PL) measurement to be $200 \mathrm{~nm}$ [52]. This value is almost comparable to that by $\mathrm{Cl}_{2}$ RIBE [64]. In comparison with this value, the damage induced into sidewalls is shallow, as shown in Section IV. The sidewall roughness was measured, as shown in Fig. 8(b), where titanium (Ti) film was used as a mask. Etch rate for $\mathrm{Ti}$ was $1 \mathrm{~nm} / \mathrm{min}$ or less. The maximum roughness $\sigma$ was $10 \mathrm{~nm}$, but locally $2 \mathrm{~nm}$ or less. It is mainly deteorirated by the edge roughness of patterned Ti film. The problem of this RIBE is the inclination of sidewalls caused by the retreat of mask edge [74]. Effective protection of mask

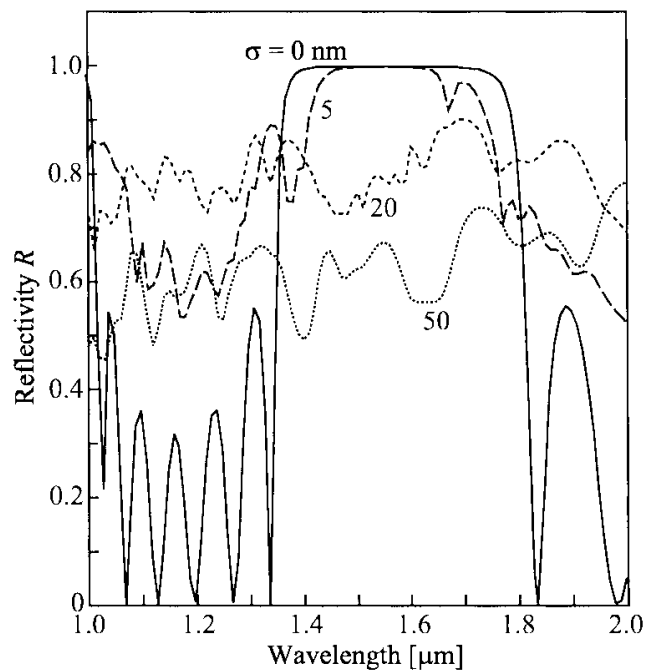

Fig. 9. Simulated reflection spectra for semiconductor/air multilayer mirrors with roughness of $\sigma$. Index of semiconductor was assumed to be 3.4.

by deposited polymers during the etching is not expected for such low gas pressure, otherwise the roughness of sidewalls is increased by the irregular deposition of polymers for a higher gas pressure. Sidewall angle against the substrate plane was typically $78^{\circ}-82^{\circ}$. This value makes it difficult to obtain a high aspect ratio over 10 and realize such an ultra-deep structure, as shown in Fig. 5 .

Selective wet etching of $\mathrm{InP}$ by $\mathrm{HCl}$ solution and that of GaInAsP by mixture of $\mathrm{H}_{2} \mathrm{SO}_{4}, \mathrm{H}_{2} \mathrm{O}_{2}$ and $\mathrm{H}_{2} \mathrm{O}$ are wellknown simple methods to to form fine structures. Especially, the former exhibits a high etch rate ratio against quaternary materials over 1000 [12]. In this paper, $1.05-\mu \mathrm{m}-Q$ and InP layers were successively grown on an InP substrate by a low-pressure metal-organic chemical vapor deposition (LPMOCVD). The top InP layer was removed using $\mathrm{HCl}: \mathrm{H}_{2} \mathrm{O}=$ 4:1 solution at $2{ }^{\circ} \mathrm{C}$. Fig. 10(a) shows atomic force microscope image of the surface just after the etching. Peculiar roughness of $10 \mathrm{~nm}$ in height and 40-200 $\mathrm{nm}$ in width may be caused by the slight etching of the $1.05-\mu \mathrm{m}-Q$ layer. Surface of a similar wafer with $1.1-\mu \mathrm{m}-Q$ layer was observed after the etching, as shown in Fig. 10(b). The roughness almost disappeared and only undulation of less than $2 \mathrm{~nm}$ in height remained. This shows the extremely flat interface of MOCVD-grown epilayers and almost perfect etch stop at the $1.1-\mu \mathrm{m}-Q$ surface. As seen from Fig. 9, a high reflectivity over 0.99 can be expected if such surfaces are utilized for multilayer mirrors. Fig. 11 shows a vertical cavity formed by the selective etching of InP. It is equipped with a pair of mirrors with $1.3-\mu \mathrm{m}-Q$ layers and air-gaps, both satisfying $\lambda_{s} / 4 n$ condition, and a $\lambda_{s} / n$-cavity containing GaInAs 5-quantum-wells. Fig. 12 shows that PL intensity from the quantum wells was enhanced to nearly seven times after the etching. Spectral width of $19 \mathrm{~nm}$ after the etching was almost independent of the excitation power. Therefore, it might be determined by the cavity resonance and the dispersion relation between emission wavelength and radiation angle (see Appendix B) as well as the numerical apperture of fiber probe used for detection. Photo-pumped lasing operation of similar air-gap vertical-cavity has been reported [48].

The anodic chemical etching is another interesting technique that realizes a 3-D structure with high aspect ratio and low 


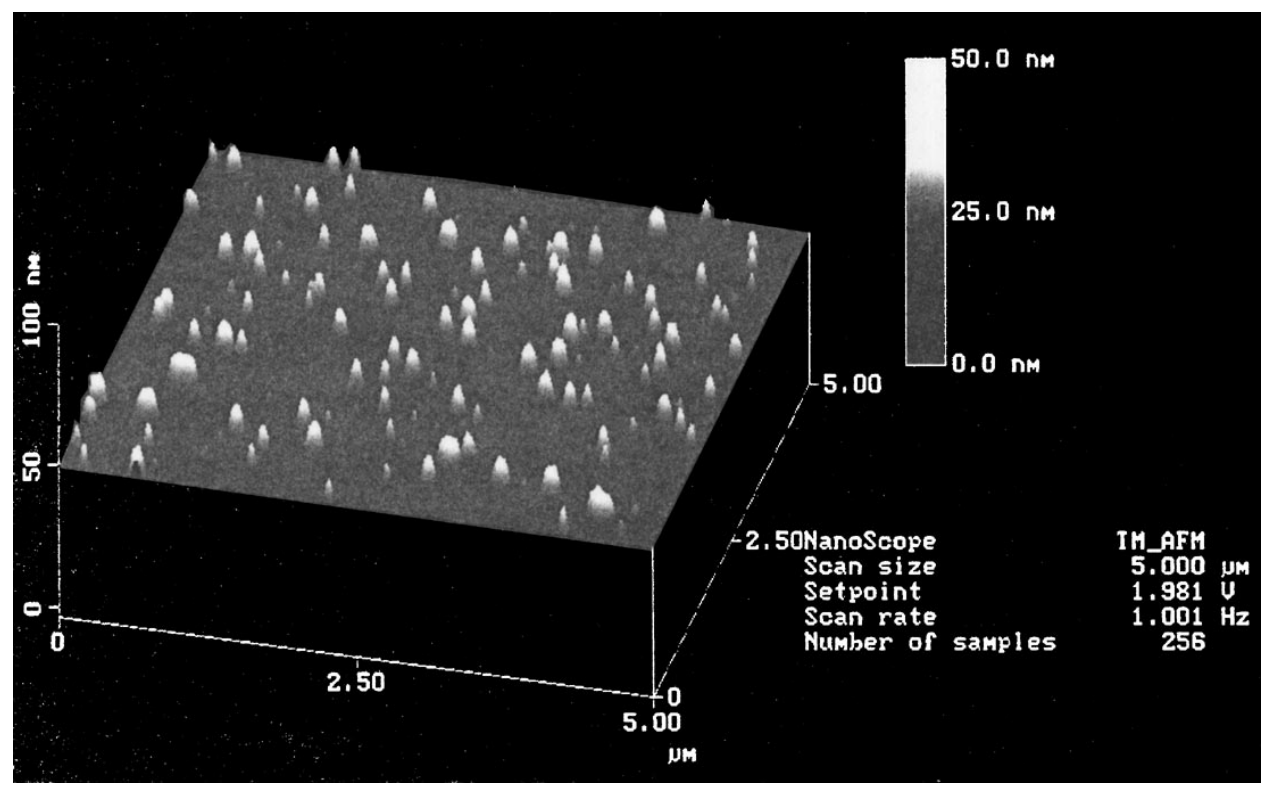

(a)

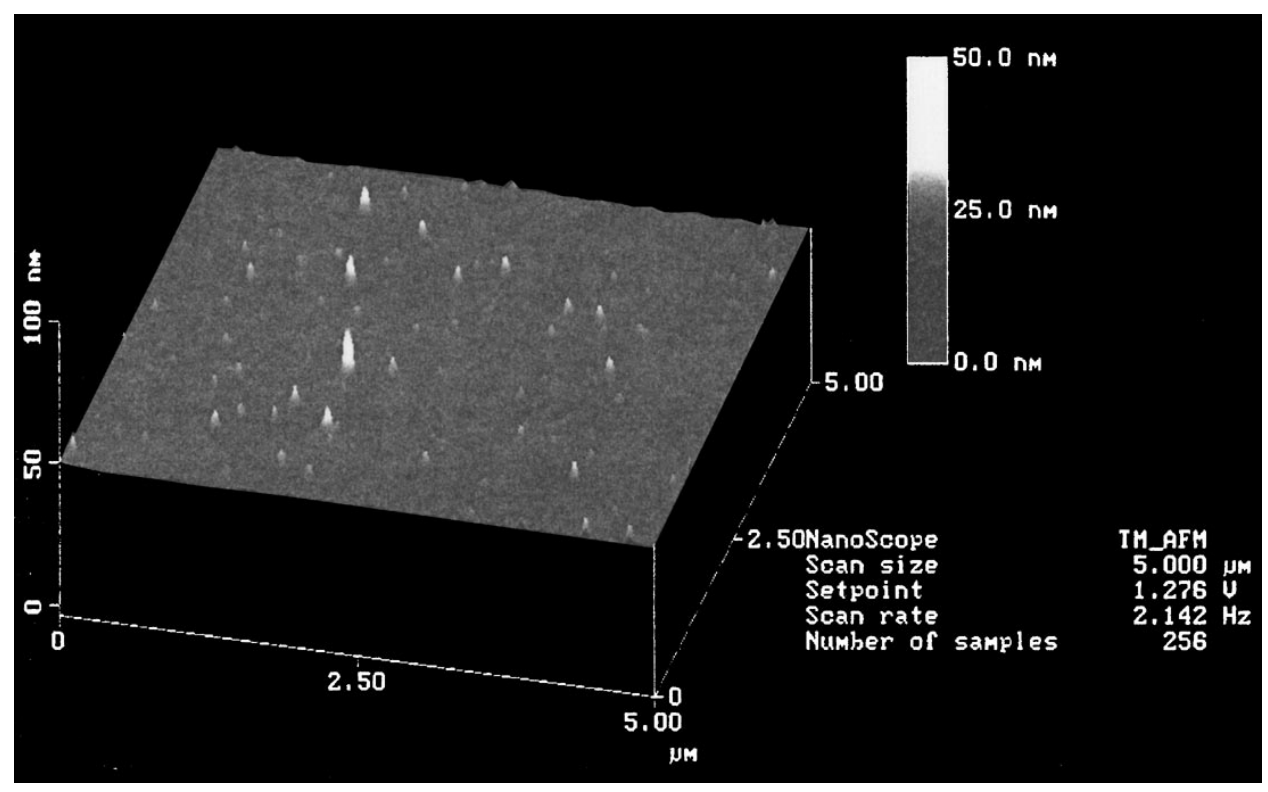

(b)

Fig. 10. Atomic force microscope image of quaternary (Q) layer surface after wet etching using $\mathrm{HCl}$ solution. (a) displays $1.05-\mu \mathrm{m}-Q$ surface and (b) $1.1-\mu \mathrm{m}-Q$ surface.

etching damage. When the surface of (111)A n-InP substrate with donnor concentration over $5 \times 10^{18} \mathrm{~cm}^{-3}$ is etched by $\mathrm{HCl}: \mathrm{H}_{2} \mathrm{O}=3: 17$ solution at RT with current injection, weak optical pumping by a fluorescent lamp and no masks covering the surface, numerous equilateral triangular holes and pillars are automatically formed [51], [70]. The typical anodization time is $3-5 \mathrm{~min}$ and current density is $10-50 \mathrm{~mA} / \mathrm{cm}^{2}$. Holes and pillars are normal to the substrate plane, and orientation of triangles is dominated by the crystal orientation. Size of triangles can be changed by the anodization time; it is of submicron order when the time is shorter than $3 \mathrm{~min}$. Even with anodization for $3 \mathrm{~min}$, the etched depth can be over $10 \mu \mathrm{m}$, and aspect ratio of $10-100$ is easily obtained. For such structures, PL intensity was almost the same before and after the anodization [51]. This may be owing to the stable crystalographic plane of exposed sidewalls. The position of triangles can be controlled by masking [70], but the shape and orientation cannot be changed without changing the wafer. One of the problem of this method is the (111)A InP substrate, which is known as a crystalographic plane not suitable for epitaxial growth. For (100) n-InP substrate, cross-hatched holes with angle of $35.6^{\circ}$ against the substrate plane appear after the same etching.

Fabrications of photonic crystals and whispering gallery mode cavities require a high aspect ratio ( $>10$ is desired), smooth surface and/or sidewall with $\sigma<5 \mathrm{~nm}$, and adaptability to arbitrary shapes. At present, no methods satisfy these requirements simultaneously. For the formation of 3-D deep structures, $\mathrm{CH}_{4}$ - $\mathrm{RIBE}$ seems to be the most promising method that exhibits sufficient performance for the latter two 


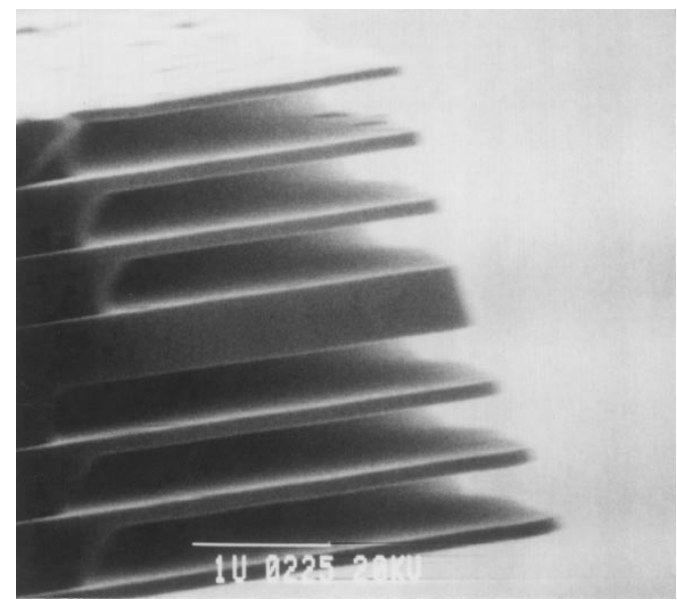

Fig. 11. SEM view of vertical-cavity constructed by GaInAsP/air-gap multilayers satisfying $\lambda_{s} / 4 n$ condition and GaInAs-GaInAsP $\lambda_{s} / n$-cavity.

requirements and has room for the improvement of aspect ratio by a multilayer etching mask.

\section{PHOTONIC CRYSTALS}

Photonic crystals are nanostructures constructed by optical atoms periodically arranged in a background medium [7]-[9], [75]-[78]. They are attracting attention because of the large potentiality for spontaneous emission control effects by the multidimensional optical reflection. However, there have been few reports on the generalized discussion and quantitative estimation of the effects due to the complicated mode behavior, which is barely examined by a long time computation. In the following, Section IV-A gives some quantitative estimation using rough approximation of modes. Likewise, there have been few reports on semiconductor structures designed for lightwave frequencies due to the difficulty of fabrication and evaluation. Sections IV-B and IV-C describe the design, fabrication, and evaluation of 2-D crystals based on GaInAsP-InP system.

\section{A. Dimensions and Spontaneous Emission Control}

One of the peculiar characteristics of photonic crystals is the photonic bandgap, which means the frequency range that inhibits the existence of modes. In photonic crystals made of semiconductor, transition spectrum overlapping with the bandgap is inhibited and the spontaneous emission rate is reduced. Fig. 13 illustrates the schematic of a photonic crystal of various dimensions, corresponding wavevector space of modes and that of spontaneous emission, which are inhibited by each photonic bandgap. The transition spectrum is expressed by a shell with radius $\omega_{s}$ and thickness $\Delta \omega_{s}$. In Fig. 13, $\omega_{s}$ is assumed to be equal to the center frequency $\omega_{g}$ of bandgap. Multilayers, as shown in Figs. 5 and 11, are kinds of 1-D crystal and the bandgap is equivalent to the stopband. However, the inhibition of light is not perfect, since radiation parallel to the multilayer plane is permitted. 2-D crystals typically consist of vertical holes or pillars formed on a substrate. Although it permits radiation normal to the substrate plane, total solid angle of inhibited radiation is much larger than that in 1D crystals. 3-D crystals consist of complicated mosaics and radiation in all directions is inhibited in the ideal case.

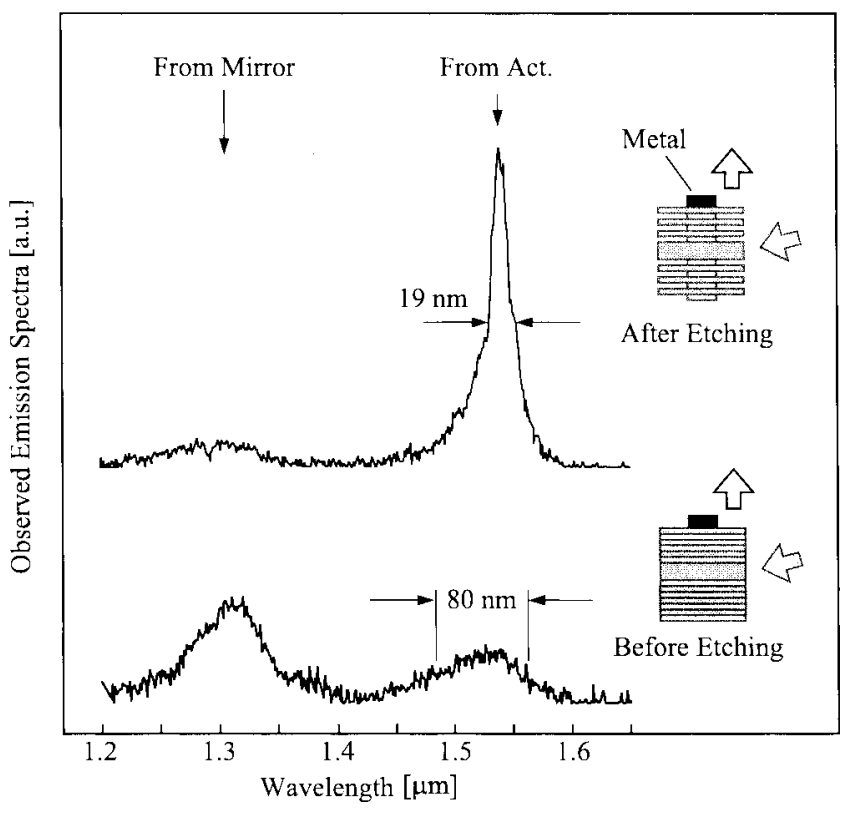

Fig. 12. PL spectra from vertical-cavities of Fig. 11.
3D
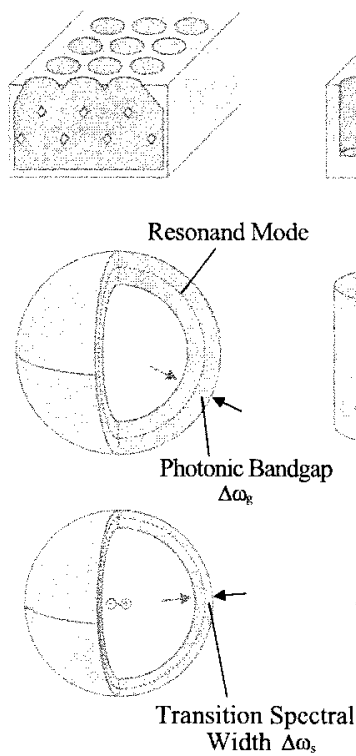

(a)
2D
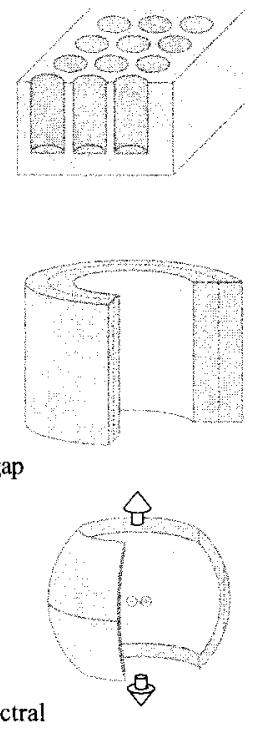

(b)
1D
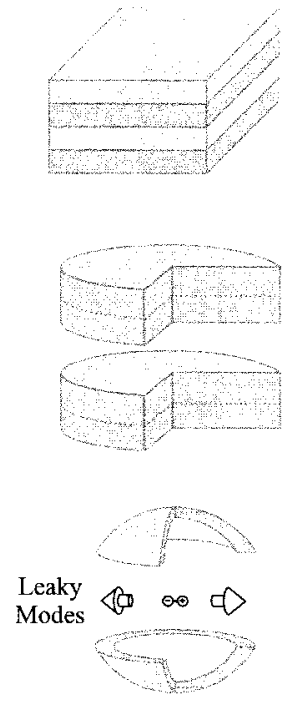

(c)
Fig. 13. (a) Schematic structures of photonic crystal of various dimensions. (b) Wave vector space inhibited by photonic bandgap. (c) Emission spectrum inhibited by photonic bandgap.

Here, leaky modes are defined as those which are not inhibited by the photonic bandgap. The simple assumption that the number of leaky modes in 1-D and 2-D crystals is proportional to the solid angle occupied by leaky modes gives the ratio $\gamma$ with normalized width of bandgap $\Delta \omega_{g} / \omega_{g}$, as shown in Fig. 14. With the expansion of $\Delta \omega_{g} / \omega_{g}$, the solid angle for leaky modes becomes small, and hence $\gamma$ is decreased. If a leaky mode is available as a laser mode, the threshold can be reduced. In 3-D crystals, the spontaneous emission energy can couple to a limited number of modes out of bandgap. As shown in Fig. 14, $\gamma$ is rapidly decreased to a very small value, once the bandgap almost overlaps with the 


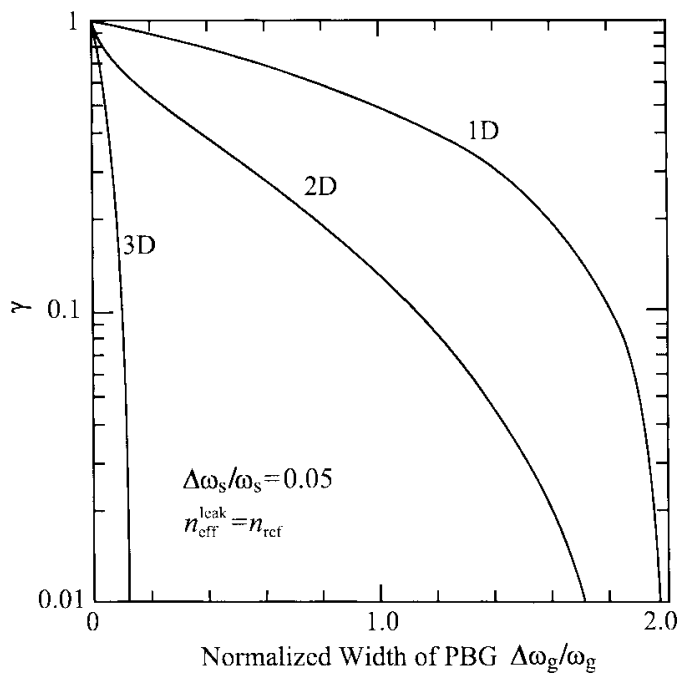

Fig. 14. Decrease of spontaneous emission rate due to inhibition of modes by photonic bandgaps.

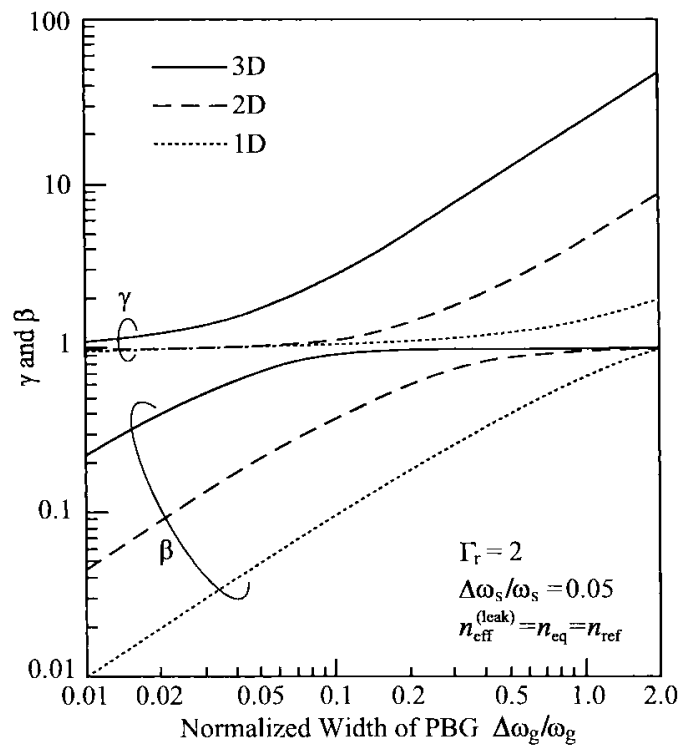

Fig. 15. Alternation ratio of spontaneous emission rate $\gamma$ and spontaneous emission coupling efficiency $\beta$ in photonic crystals. Since they are approximately obtained by neglecting change of equivalent index, result for 1-D crystals is slightly different from that of Fig. 36.

transition spectrum. For this case, it seems difficult to utilize a leaky mode as a laser mode.

Irregular optical atoms put into photonic crystals produce resonant modes whose fields localize around the atoms and resonant frequencies locate inside the photonic bandgap [8], [9]. Let me suppose an irregular atom of nearly $\left(\lambda_{s} / n\right)^{3}$ in volume, which contains the active region with a high refractive index. Antinode of localized field efficiently overlaps with the active region, and large $\Gamma_{r}$ is realized when the active region is much smaller than the irregular atom and is located at the center of the atom. Under this condition, the resonant mode overlaps with the transition spectrum in the wavevector space, as denoted by dotted lines in Fig. 13. The resonant mode in 1-D crystals has discrete wavevectors in the vertical direction, but continuous ones in the lateral directions. There exists a dispersion relation between resonant frequency and radiation

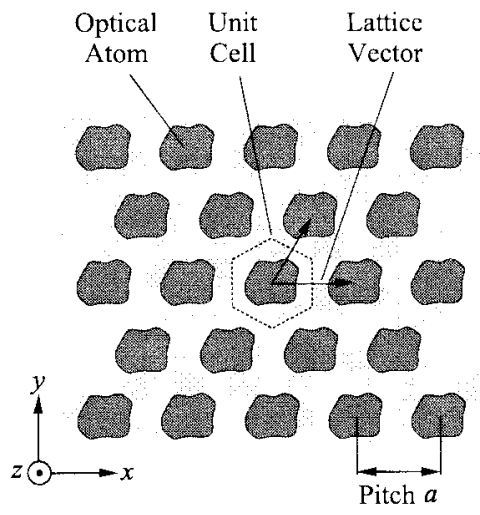

(a)

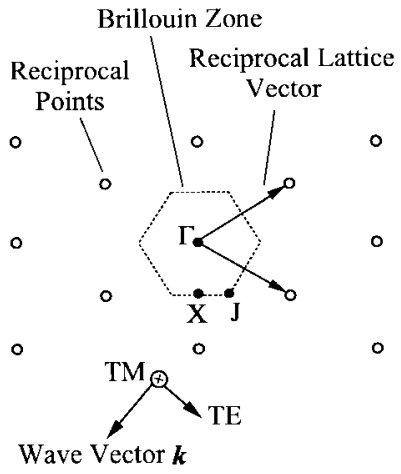

(b)

Fig. 16. (a) Triangular lattice and (b) corresponding reciprocal lattice in 2-D crystal with coordinate system and definition of TE and TM polarizations in this paper.

direction. Emission coupled to this mode is radiated toward upper and lower directions with solid angle $2 \pi \Delta \omega_{s} / \omega_{s}$.

Almost precise numerical calculation of spontaneous emission rate for definite 1-D crystals has been performed [22], but not for 2-D and 3-D due to the long time computation for the volume of localized field of resonant mode and $\Gamma_{r}, n_{\mathrm{eq}}$ and $n_{\text {eff }}$ for all modes including leaky modes. However, normalized width of bandgap $\Delta \omega_{g} / \omega_{g}$ in each photonic crystal is roughly approximated by the mode interval in the ideal cavity discussed in Section II (see Appendix B). The change of $\gamma$ is outlined for each crystal, as shown in Fig. 15. $\gamma$ can be larger than 1 with the expansion of $\Delta \omega_{g} / \omega_{g}$ in 2D and 3-D crystals. A summary of many photonic band calculations showed that the maximum $\Delta \omega_{g} / \omega_{g}$ for 3-D crystals made of semiconductor and air is nearly 0.3 [79]. For this value, $\gamma_{3}=8$ is estimated from Fig. 15. For 2-D crystals constructed by columns, $\Delta \omega_{g} / \omega_{g}=0.46$ is predicted for a specified polarization, as shown later, and thus $\gamma_{2}=2$. In Fig. $15, \Gamma_{r}$ and $\Delta \omega_{s} / \omega_{s}$ are assumed to be 2 and 0.05 , respectively, considering quantum film active region with a standard transition spectrum from GaInAsP-InP observed at room temperature. If $\Gamma_{r}$ is increased and $\Delta \omega_{s} / \omega_{s}$ is decreased by quantum-dot or quantum-wire active region, $\gamma$ is increased to $10-20$ for 3-D crystals.

$\beta$ is defined as the coupling efficiency of spontaneous emission energy into resonant modes with continuous wavevector [15], [16]. Using the same assumption as for $\gamma$, the change of $\beta$ is also examined, as shown in Fig. 15. The spontaneous emission factor $C$ is given for an absolute discrete mode 

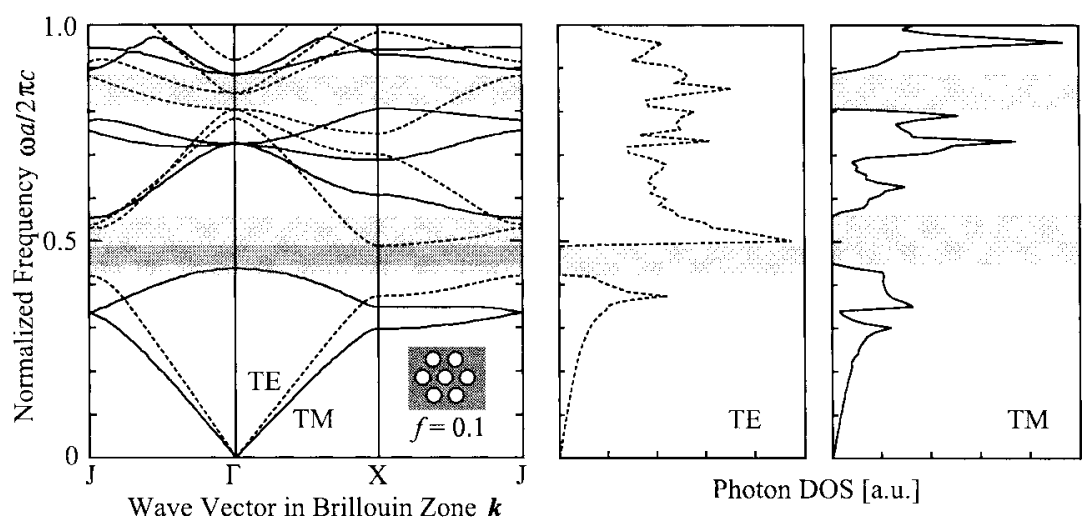

(a)
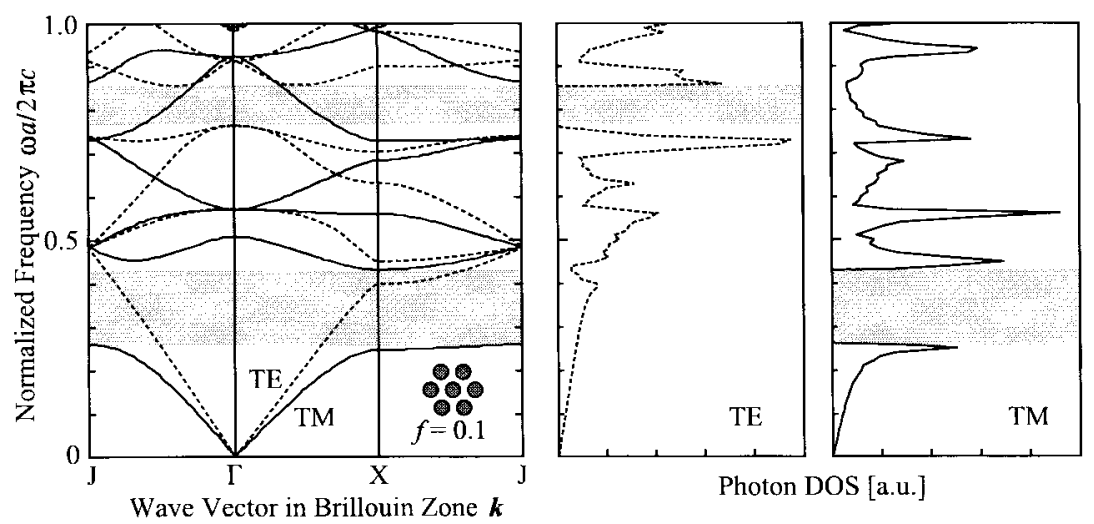

(b)

Fig. 17. Photonic bands and photon DOS for circular atoms and triangular lattice. Assumed refractive index for semiconductor denoted by shadowed region in insets was 3.46, which is that of InP at band edge. Results for this index is applicable to those made of various III-V compounds with small error.

defined by 3-D boundary conditions. In an ideal 3-D crystal with one irregular atom, $C$ is equivalent to $\beta$ if the resonant mode has no polarization degeneracies, and thus $C$ can be almost 1 with $\Delta \omega_{g} / \omega_{g}>0.1$. To discuss the $C$ factor for 1-D crystals, they need some side mirrors. The simplest mirror is the sidewall formed by etching a planar 1-D crystal into a post shape. It has been shown that $C$ over 0.1 is possible in an airpost GaAs-AlGaAs VCSEL when the post width is reduced to $\lambda_{s} / n$ [22]. Similarly, upper and lower mirrors are necessary for 2-D crystals. One of the method imagined is to form holes or columns into a planar 1-D crystal. For this case, a 3-D localized field can be defined and $C \sim \beta$ may be possible.

\section{B. Design of Semiconductor 2-D Crystals}

2-D crystals were designed from photonic band diagrams calculated by solving the eigenvalue equation for vector waves [80]-[83] with the numerical Fourier transform of atom shapes. Schematic top view of a 2-D crystal with the triangular lattice [84], [85] and the corresponding reciprocal lattice are illustrated in Fig. 16. For wavevectors inside the 2-D plane, polarization parallel to the 2-D plane (TE polarization) and that perpendicular to the 2-D plane (TM polarization) can be considered individually, since eigenvalue equations are separated for these polarizations. Typical photonic band diagrams for circular atoms are shown in Fig. 17. The shadowed and bright regions in the insets indicate a semiconductor and air, respectively. The filling fraction $f$ of semiconductor is fixed to 0.1 . For circular holes formed into semiconductor, the overlap of photonic bandgaps for two polarizations is observed, which absolutely inhibits the spontaneous emission inside the 2-D plane. Normalized width $\Delta \omega_{g} / \omega_{g}$ of the absolute bandgap is 0.1 . For semiconductor columns (or circular pillars), $\Delta \omega_{g} / \omega_{g}$ of the lowest bandgap for TM polarization is as wide as 0.46 . As seen in Fig. 15, $\beta>0.9$ is expected for this bandgap if a resonant mode is produced by an irregular atom and the polarization is restricted to TM by an anisotropic orientation of dipoles in quantum-wells. Photon density of states (DOS) for each polarization is also obtained by integrating $d k / d \omega=$ $n_{\text {eff }} / c$ inside the Brillouin zone of Fig. 16. It repeats increasing and decreasing with the change of $\omega a / 2 \pi c$, and sharp peaks appear at band edges.

Experimentally, dry etching techniques allow arbitrary shapes of optical atom, but chemical etchings and selective area growths [53], [56] specify shapes depending on the crystallographic plane of semiconductors. To simulate experimental shapes, the distribution of photonic bandgap for various shapes with various filling fractions $f$ was examined, as shown in Fig. 18. Some of photonic bandgaps of $\Delta \omega_{g} / \omega_{g}<$ 0.05 were omitted. In general, a structure constructed by holes is likely to exhibit TE bandgaps, while that by pillars TM bandgaps. They are explained to be originated from the abrupt change of displacement field in continuous structures like holes and that of magnetic field in discrete structures like pillars, respectively, at bandgaps [75]. The absolute bandgap is obtained for circular holes with $0.09<f<0.2$, and $\Delta \omega_{g} / \omega_{g}$ is maximally 0.15 for $f=0.13$. For columns, several wide 


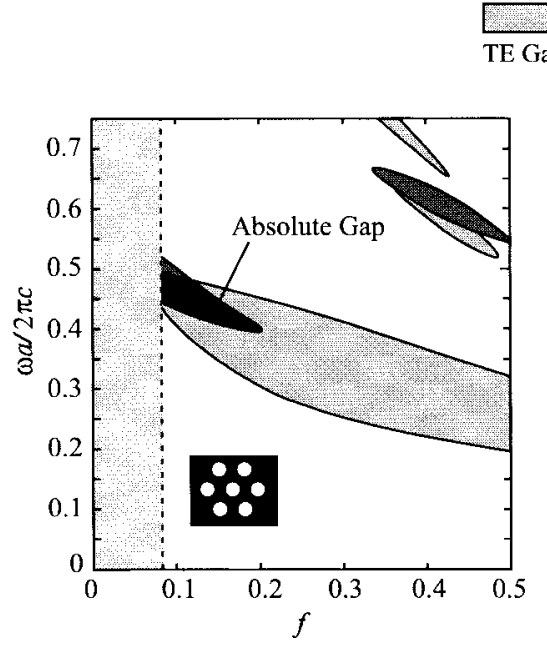

(a)

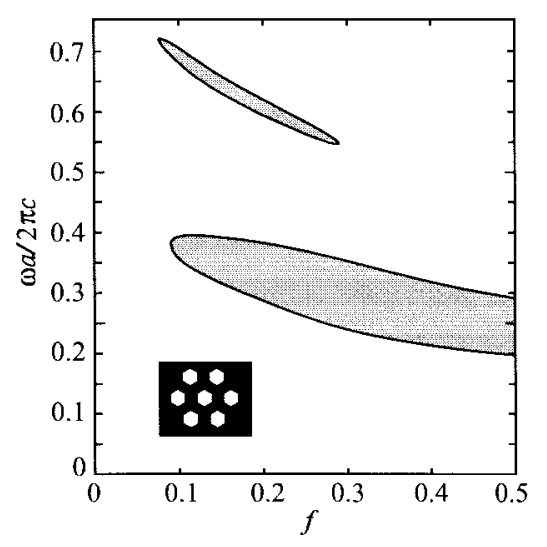

(c)
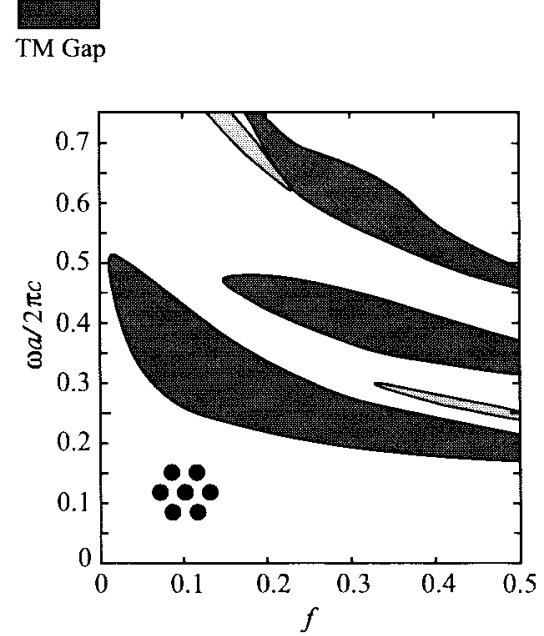

(b)

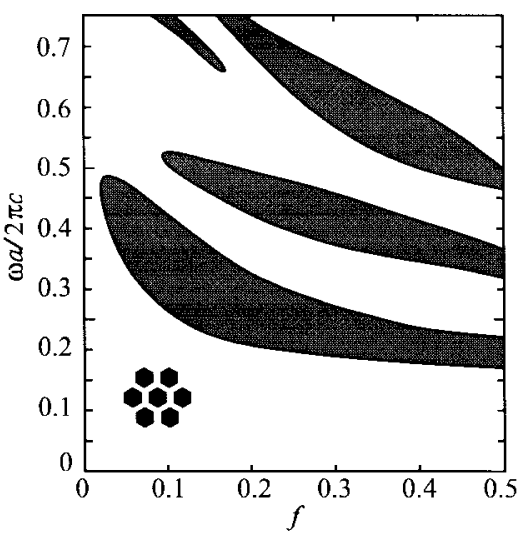

(d)

Fig. 18. Dependence of photonic bandgap on filling fraction $f$. Assumed shape of optical atoms is illustrated in each inset. Dark region indicates semiconductor whose refractive index is 3.4. Bandgaps for $f<0.09$ is not calculated in (a) and that for $f<0.13$ in (e), since air atoms cannot be circular and square under the conditions, respectively.

TM bandgaps and narrow TE bandgaps are obtained for a wide rage of $f$. For hexagonal atoms, the distribution of bandgap is similar to that for circular atoms. However, the absolute bandgap is not observed, since hexagonal holes do not separate the semiconductor with any fraction $f$. For square and triangular pillars, the distribution of TM bandgaps is similar to that for columns. For square holes, TE and TM bandgaps exist simultaneously but do not overlap with each other.

As illustrated in Fig. 19, four types of emitting device based on 2-D crystals with circular atoms are supposed. Structures of Fig. 19(a) and (b) are equipped with a small hole and a large column at the center, respectively, to maintain a resonant mode. Spontaneous emission coupled into this mode is radiated in the lateral directions. The radiation direction will be further restricted by putting the irregular atom out of center or removing some atoms locating on the way from the irregular atom to the edge of the crystal. The latter one seems to be more advantageous for the collection of light power, since the atom-removed region acts as a low loss waveguide [83]. In Fig. 19(c) and (d), no irregular atoms are introduced so that the lateral emission is inhibited by photonic bandgaps. It is effective in the enhancement of external efficiency in surface emitting LED's and the reduction of threshold current in VCSEL's by the reduction of spontaneous emission rate.
Since columns with the triangular lattice has the imperfection in TE bandgaps, the structure (d) employs the hexagonal lattice, which also exhibits an absolute 2-D bandgap [75].

Let me check the actual size of 2-D crystals exhibiting photonic bandgaps at lightwave frequencies. Emission wavelengths $\lambda_{s}$ of 1.55 and $0.88 \mu \mathrm{m}$ are assumed for GaInAsP-InP and GaAs-AlGaAs systems, respectively. In Fig. 18(a), the absolute bandgap for circular holes lies around $\omega_{g} a / 2 \pi c=$ 0.45 with $f=0.13$. Corresponding pitch $a$ and diameter of holes are 0.698 and $0.683 \mu \mathrm{m}$ for $\lambda_{s}=1.55 \mu \mathrm{m}$, respectively, and 0.396 and $0.388 \mu \mathrm{m}$ for $\lambda_{s}=0.88 \mu \mathrm{m}$, respectively. They are honeycomb structures in which only $2.1 \%$ of the pitch is remained for the minimum thickness of semiconductor walls between holes; it is as narrow as $15 \mathrm{~nm}$ and $8 \mathrm{~nm}$ for those wavelengths, respectively. In Fig. 18(b), the lowest TM bandgap for columns lies around $\omega_{g} a / 2 \pi c=0.34$ with $f=$ 0.1 . Corresponding pitch $a$ and diameter of columns are 0.527 and $0.179 \mu \mathrm{m}$ for $\lambda_{s}=1.55 \mu \mathrm{m}$, respectively, and 0.299 and $0.099 \mu \mathrm{m}$ for $\lambda_{s}=0.88 \mu \mathrm{m}$, respectively. To operate 2-D crystals properly as expected, height of those structures must be much larger than pitch $a$. The minimum aspect ratio required for the thinnest semiconductor walls between holes is $48: 1$, and for columns 3:1. The former requirement seems to be difficult for GaInAsP-InP system by any dry etchings at present. 


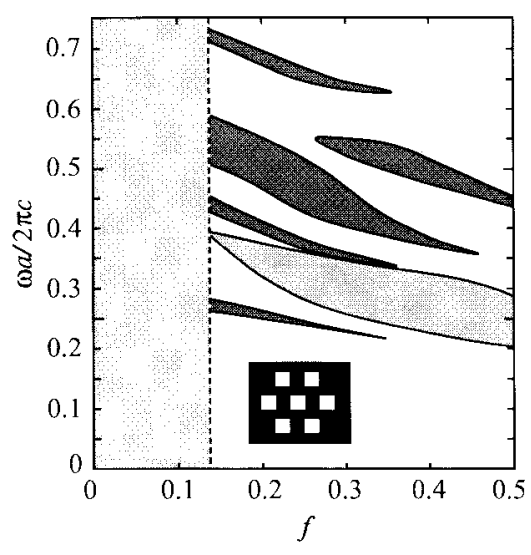

(e)

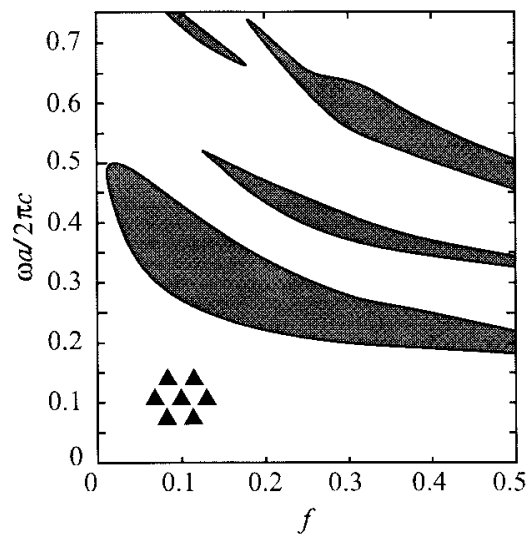

(g)

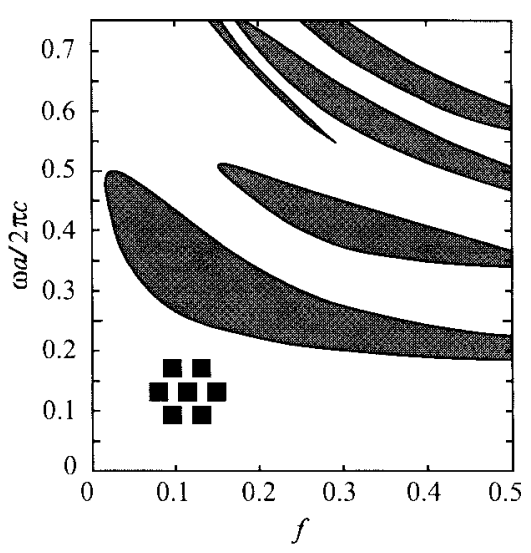

(f)

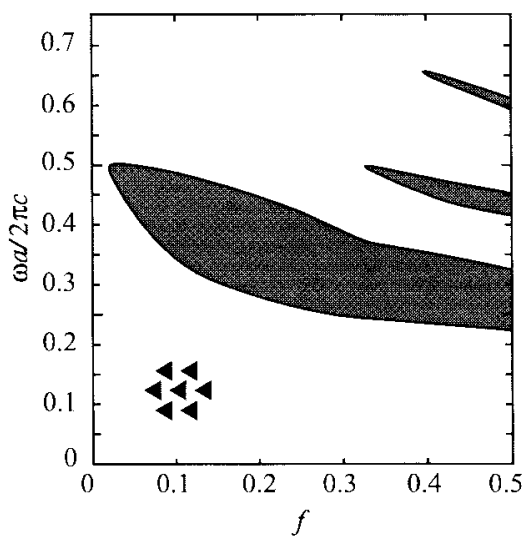

(h)

Fig. 18. (Continued.) Dependence of photonic bandgap on filling fraction $f$. Assumed shape of optical atoms is illustrated in each inset. Dark region indicates semiconductor whose refractive index is 3.4. Bandgaps for $f<0.09$ is not calculated in (a) and that for $f<0.13$ in (e), since air atoms cannot be circular and square under the conditions, respectively.

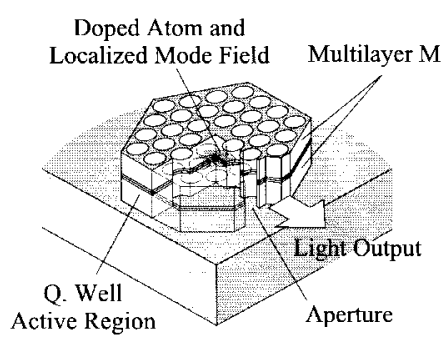

(a)

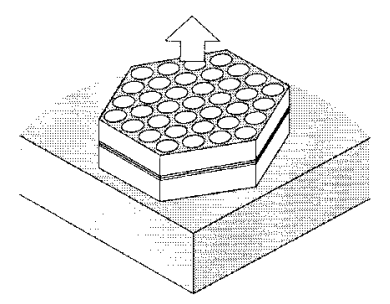

(c)

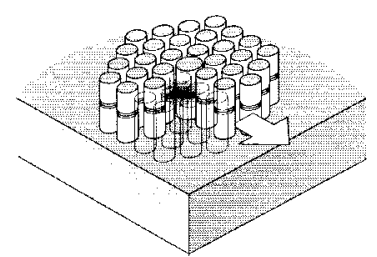

(b)

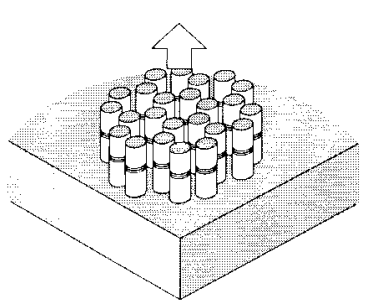

(d)

Fig. 19. Schematics of emitting device based on 2-D crystals with circular atoms.

\section{B. Fabrication and Evaluation of GaInAsP-InP 2-D Crystals}

In this study, the arrangement of columns with the triangular lattice was employed as a structure of 2-D crystal to minimize the fabrication difficulty. GaInAsP compressive-

strained multiple-quantum-well (CS-MQW) and gradientindex separate-confinement-heterostructure (GRIN-SCH) were grown on InP substrate by an LP-MOCVD. Each thickness of well layers was 3-4 nm and that of $1.2-\mu \mathrm{m}-Q$ barrier layers $10 \mathrm{~nm}$. The strain was $1 \%$, and the number of wells was four. Total thickness of active layer including 50-nm-thick 1.2- $\mu \mathrm{m}$ $\mathrm{Q}$ guide layers and 30-nm-thick 1.1- $\mu \mathrm{m}-\mathrm{Q}$ GRIN layers was nearly $0.2 \mu \mathrm{m}$. The peak emission wavelength was $1.545 \mu \mathrm{m}$ at RT. For the formation of columns, both $\mathrm{Cl}_{2}$-based RIBE and $\mathrm{CH}_{4}$-based RIBE were tested. However, the loading effect in the former method was serious and the sidewall roughness was over $50 \mathrm{~nm}$ for the dense pattern of columns. Therefore, only fabrication process by $\mathrm{CH}_{4}$-based RIBE is described below. First, Ti film of 40-70 nm in thickness was evaporated on the wafer as a mask. On this film, SAL601 resist of circular dot patterns was formed using a standard EB lithography system with a tungsten filament and acceleration voltage of $20 \mathrm{kV}$. The filling fraction $f$ was maintained to 0.1 for various dot diameters. Through the patterns, the Ti was patterned by RIBE with $\mathrm{CF}_{4}$ gaseous source, and successively the epilayers were etched with mixture of $\mathrm{CH}_{4}, \mathrm{H}_{2}$, and $\mathrm{Ar}$ under the condition described in Section III. Formed columns have smooth sidewalls, as seen in Fig. 20. Fluctuation of diameter in the smallest columns was due to the resolution limit of the lithography system rather than the RIBE. The sidewall angle of the smaller two was over 85 degrees, while that of 


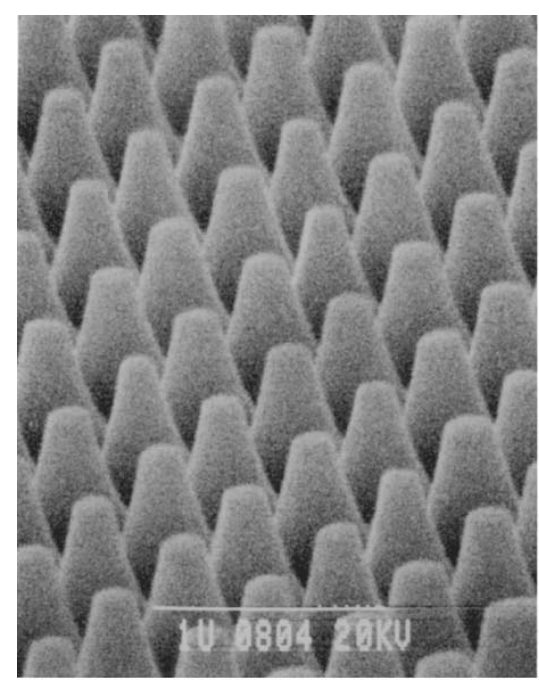

(a)

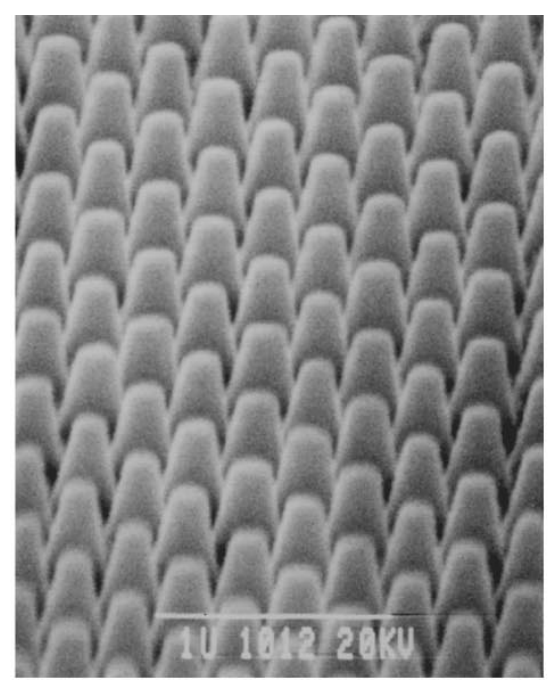

(b)

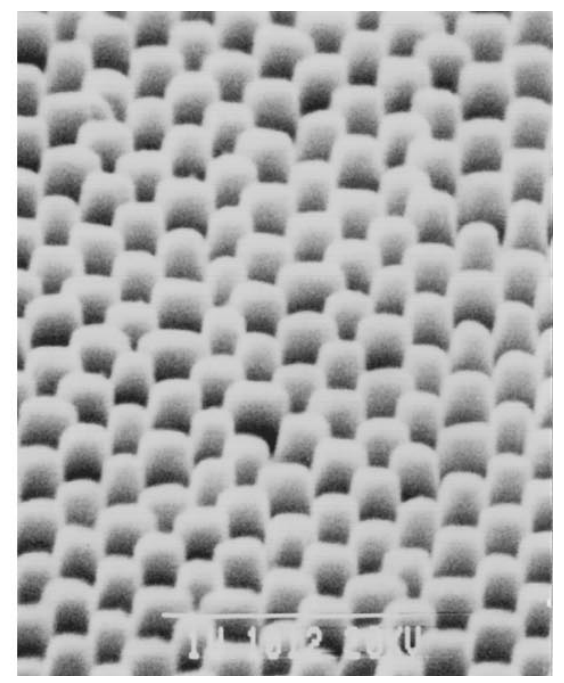

(c)

Fig. 20. SEM views of 2-D crystal constructed by GaInAsP-InP columns. Diameter and pitch are: (a) 0.25 and $0.75 \mu \mathrm{m}$; (b) 0.2 and $0.625 \mu \mathrm{m}$; and (c) 0.15 and $0.45 \mu \mathrm{m}$, respectively. Height was $0.6-0.8 \mu \mathrm{m}$.

the larger two was $72^{\circ}-78^{\circ}$. This difference comes from the slight difference of Ti mask edge. The height of columns was $0.6-0.8 \mu \mathrm{m}$, and the maximum aspect ratio obtained was 4 . PL spectra were measured before and after the etching at room temperature from the direction almost normal to the substrate surface. Here, the pump wavelength was $830 \mathrm{~nm}$ and pump density $1 \mathrm{~kW} / \mathrm{cm}^{2}$. It was observed that PL intensity multiplied by fraction $f$ was degraded to $1 / 2,1 / 5$, and $\ll 1 / 10$ by reducing the diameter of columns to $0.7,0.4$, and $<0.2 \mu \mathrm{m}$, respectively. The RIBE damage depth into sidewalls is estimated to be less than $100 \mathrm{~nm}$. Upon wet etching for $6 \mathrm{~s}$ using $\mathrm{HCl}: \mathrm{H}_{2} \mathrm{O}=4: 1$ solution at $2{ }^{\circ} \mathrm{C}, 0.4-\mu \mathrm{m}$-diameter columns were deformed to a mushroom shape, as shown in Fig. 21, but the PL intensity multiplied by $f$ was recovered to almost the same level or even stronger as that from the as-grown wafer. The stronger emission after the wet etching seems to be due to the mushroom shape, which radiates and scatters the emission more efficiently than flat surface of the as-grown wafer [7].

For formed columns with diameter of 0.35 and $0.40 \mu \mathrm{m}$, polarization characteristics of PL intensity was measured after the wet etching in the $\Gamma-\mathrm{X}$ direction inside the 2-D plane, as shown in Fig. 22. Here, the pump condition was the same as mentioned above. The emission from the edge was collimated and focused into a multimode fiber by a pair of lenses. The polarization of the emission was analyzed by a polarizer inserted between the lenses. The emission from as-grown wafer was TE-polarized, while that from the formed structures was TM-polarized. The TE-polarized emission seems to be due to the anisotropic dipole orientation caused by the splitting of valence bands in the CS-MQW [87]. The TM-polarized emission can be explained as the effect of photonic crystal. Fig. 23 shows the photon DOS calculated for the formed structures, where the mushroom shape was taken into account as the gradient-index distribution of equivalent index inside the 2-D plane [88]. As seen in Fig. 23, the structure with diameter of $0.4 \mu \mathrm{m}$ almost satisfies a TE photonic bandgap condition. The structure with diameter of $0.35 \mu \mathrm{m}$ does not overlap with any bandgaps, but it rather overlap with a small peak of photon DOS for TM polarization.

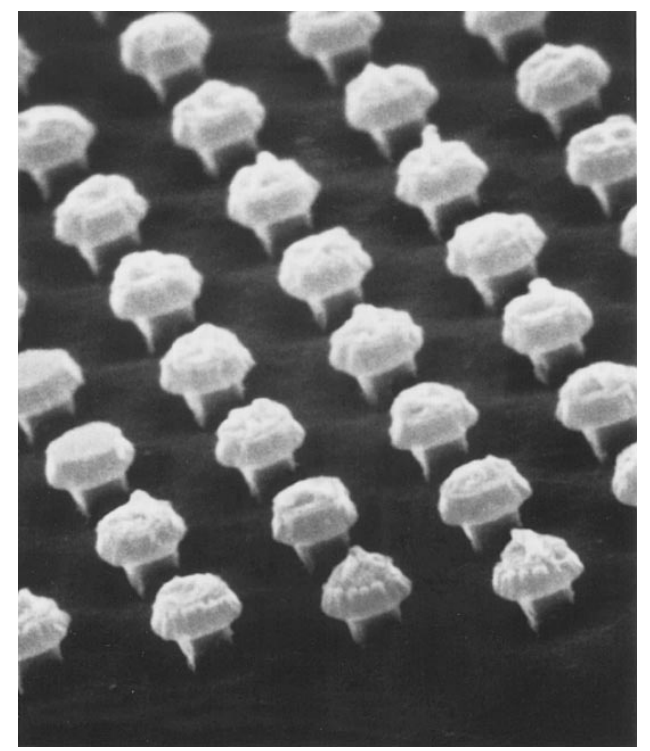

Fig. 21. Mushroom shape observed after wet etching for $4-\mu \mathrm{m}$-diameter columns.

The measurement of transmission spectra is effective for clearer evaluation of photonic bandgap [8]. However, it has never been performed for semiconductor 2-D crystals at lightwave frequencies, since in-plane transmission measurement is difficult for such small structures. In this study, the equivalent transmission measurement was performed to avoid this difficulty. Various columns were prepared on the same wafer, which satisfied $f=0.1$, i.e., $0.3-0.5 \mu \mathrm{m}$ in diameter and $0.9-1.5 \mu \mathrm{m}$ in pitch. The height was increased to $1.5 \mu \mathrm{m}$ by adding a transfer process of patterns to the Ti mask from chromium film formed by a liftoff technique. To avoid the mushroom shape, no wet etchings were performed after the RIBE. The fluctuation of diameter was suppressed to less than $3 \%$, but the inclination of sidewalls was not improved. Setup for the transmission measurement is illustrated in Fig. 24. As a light source, a large square region neighboring with the columns was used, which was not removed by the RIBE. 


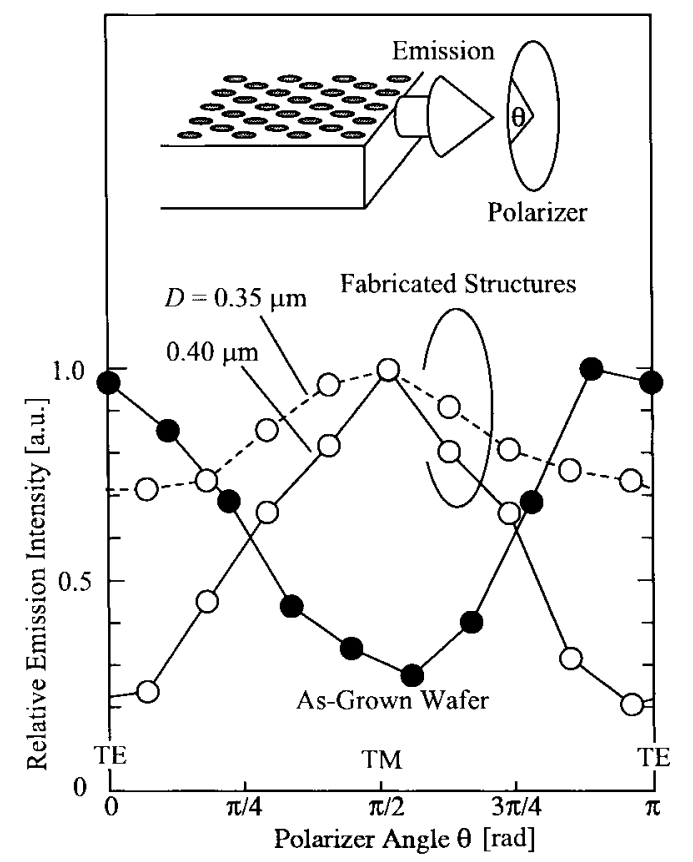

Fig. 22. Polarization characteristics of emission from as-grown wafer and fabricated structures with diameter $D$.

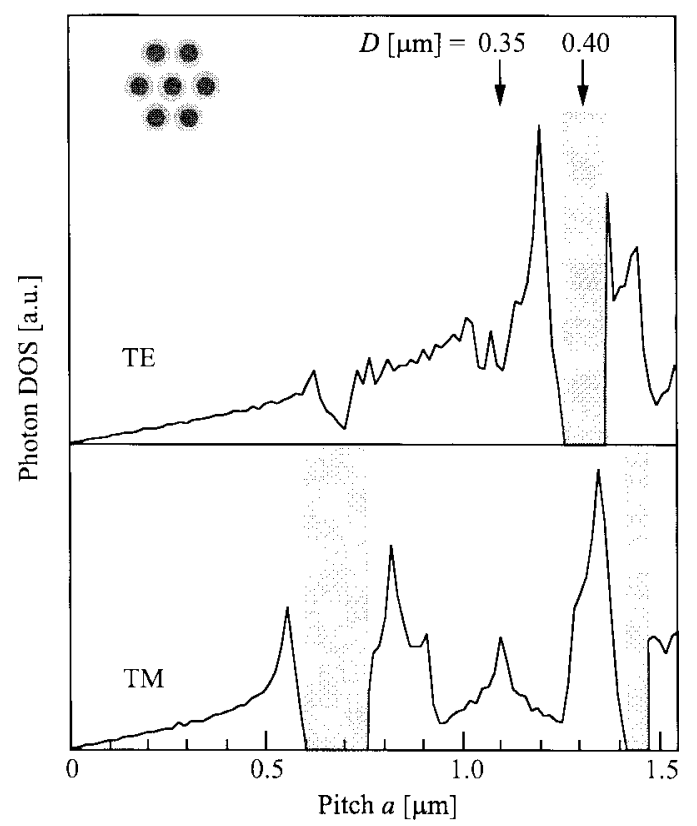

Fig. 23. Photon DOS calculated for mushroom structures formed by RIBE and slight wet etching.

This region was photopumped from the top of the wafer. The emission in the lateral direction was directly detected by a single mode fiber from a distant position and analyzed by an optical spectrum analyzer after passing through the columns. Lenses and polarizers were not inserted between the wafer and the fiber to detect sufficient light power and to minimize the variation of detecting condition. By repeating the measurement for various columns and plotting all the measured spectra on the axis of normalized frequency $\omega a / 2 \pi c$, an equivalent transmission spectrum was obtained as an envelope of the spectra. Such measurements were performed in both $\Gamma-X$ and $\Gamma-\mathrm{J}$ directions. The results are summarized in Fig. 25. Here,

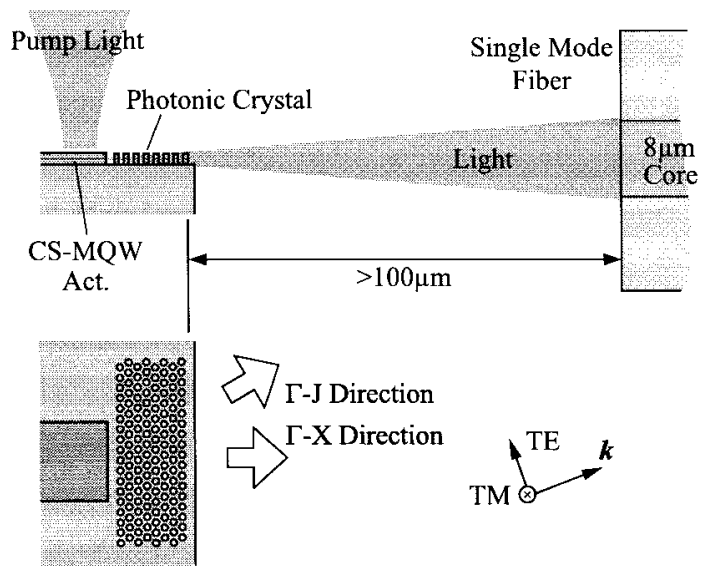

Fig. 24. Measurement setup of equivalent transmission spectra for 2-D crystal by columns.

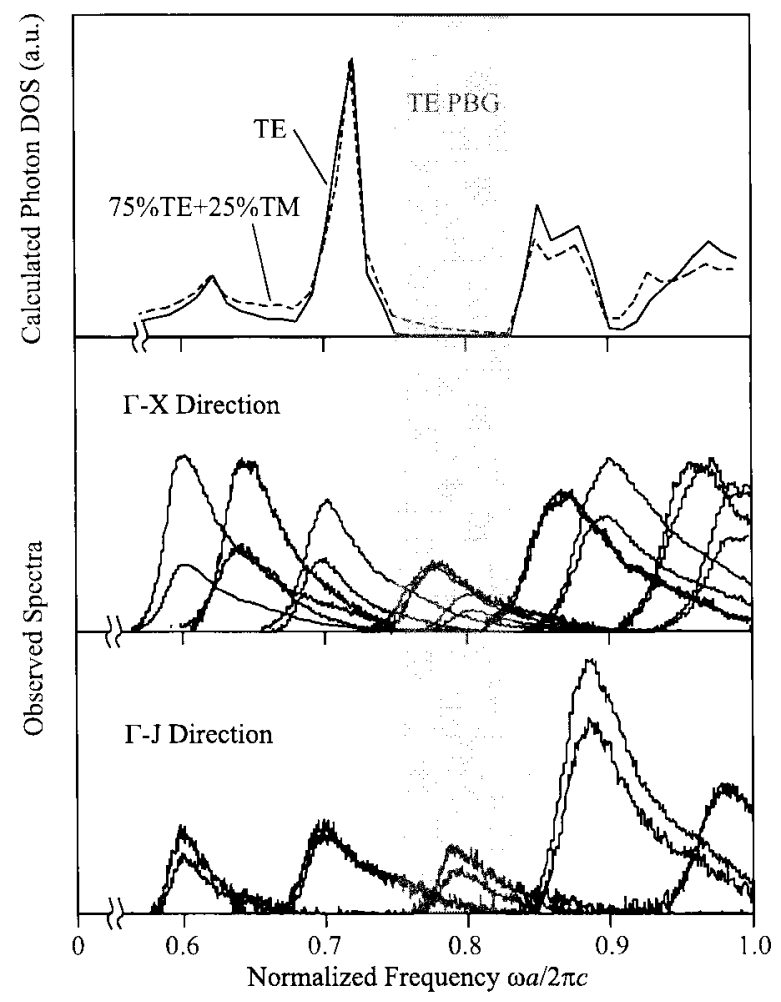

Fig. 25. Calculated photon DOS and observed transmission spectra.

calculated photon DOS for TE polarization and that for the sum of $75 \% \mathrm{TE}$ and $25 \% \mathrm{TM}$ are also plotted for comparison. The latter polarization ratio simulates the polarization anisotropy observed for the as-grown wafer. Theoretically, a TE bandgap lies around $\omega a / 2 \pi c=0.8$. In $\Gamma-\mathrm{X}$ direction, spectra almost overlapping with the bandgap showed nearly $-4 \mathrm{~dB}$ decrease of transmission intensity. Although it was ambiguous in $\Gamma-\mathrm{J}$ direction, at least $-1 \mathrm{~dB}$ decrease could be recognized at the same frequency range. It was also observed that the frequency range of lowtransmission intensity blue-shifted and the decrease of transmission intensity at the bandgap became more and more ambiguous when the fiber was slightly tilted toward upper direction. This seems to be influenced by the sidewall inclination of columns.

As seen from the experimental result, some light scattering and diffraction effects must be existing in formed columns. 


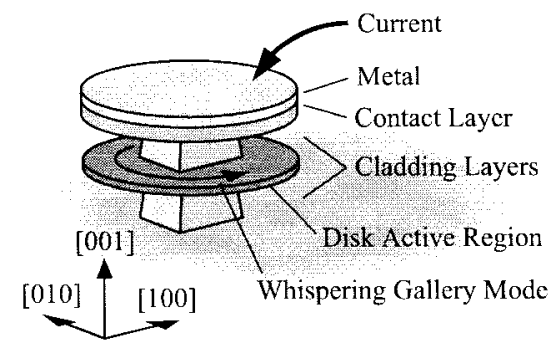

Fig. 26. Schematic of microdisk injection laser.

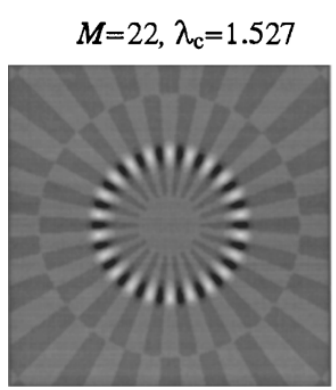

$M=17, \lambda_{c}=1.595$

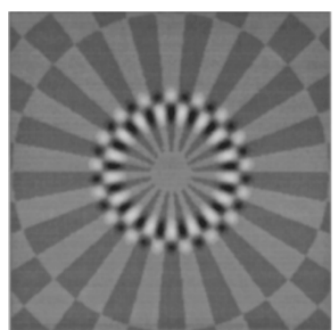

$M=21, \lambda_{\mathrm{c}}=1.590$

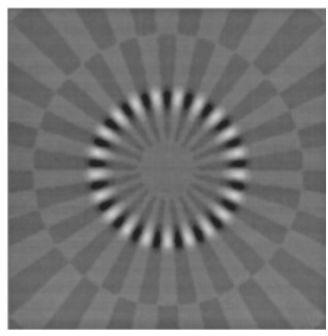

$M=14, \lambda_{\mathrm{c}}=1.587$

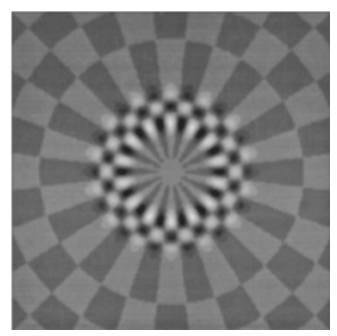

Fig. 27. Magnetic field of resonant modes in microdisk of $5 \mu \mathrm{m}$ in diameter. It is displayed by shaded drawing.

However, much effort is necessary to achieve perfect inhibition of spontaneous emission at photonic bandgaps and a high- $Q$ resonance applicable to lasers by fully uniform, precise and smooth structures. As discussed in Section III, key points are sidewalls normal to the substrate plane, which may be obtained by the further improvement of etching mask.

\section{Microdisk CAVITIES}

In compared with photonic crystals, whispering gallery mode cavities have very simple geometries such as sphere, disk, column and ring, and easily realize a high $Q$ resonance by the total internal reflection in all directions. Especially, disk cavities have already achieved extreme size reduction and low-threshold lasing operation by current injection. The following discussion focuses on this type. Other cavities are considered to have qualitatively the same characteristic as those for disk cavities. First, the relation among spontaneous emission control effects, cavity $Q$ and cavity size is discussed. Next, fabrication and evaluation of GaInAsP-InP disk lasers are presented.

\section{A. Resonant Modes and Spontaneous Emission Control}

Fig. 26 illustrates the schematic of a disk laser. The disk active region is supported by upper and lower pedestals. By injecting current from the top contact, carriers diffuse to the disk edge and produce the optical gain. The disk edge exposed to the air consists of optical waveguide in the vertical

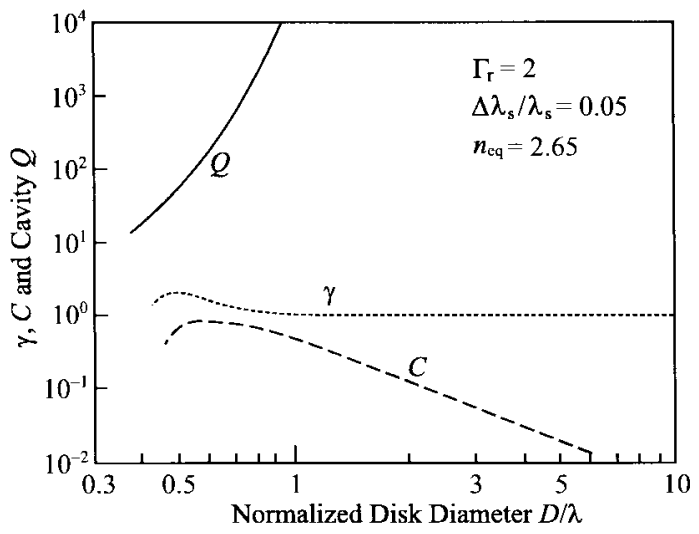

Fig. 28. Dependence of spontaneous emission rate, spontaneous emission factor and cavity $Q$ on normalized disk diameter.

direction. The lasing oscillation occurs by whispering gallery modes rounding inside the waveguide. Such modes were approximately calculated by a simple 2-D model, in which a circular region with equivalent index $n_{\mathrm{eq}}$ of the waveguide is surrounded by an outer region with index of 1 . The variable separation method assumed in this model may give some amount of error, since the wavevector inside the waveguide have a significant component in the vertical direction. I expect this model to explain some qualitative behavior of whispering gallery mode. The equivalent index, resonant wavelength and mode field distribution inside the 2-D plane for mode order $M$ were numerically obtained by simultaneously solving guidedwave equation and axially symmetric wave equation with boundary conditions at disk center, edge and infinite distance. Fig. 27 shows some examples of magnetic field distribution in a disk with $5 \mu \mathrm{m}$ in diameter, $0.2 \mu \mathrm{m}$ in thickness and 3.4 of refractive index. Here the electric field vector is fixed inside the 2-D plane, since it well simulates the polarized spontaneous emission in CS-MQW used in the experiment. The equivalent index $n_{\mathrm{eq}}$ was calculated to be $2.6-2.75$ for resonant wavelengths ranging from 1.5-1.65 $\mu \mathrm{m}$. Fig. 27(a) and (b) display typical whispering gallery modes. The wavelength difference of these modes is $63 \mathrm{~nm}$ and this value is increased by reducing the disk diameter. Fig. 27(c) and (d) display lower order modes, which have resonant wavelengths close to that of (b) and mode maxima rounding relatively inside the disk.

Using (5) and (7), $\gamma$ and $C$ are estimated with normalized disk diameter $D / \lambda_{c}$, as shown in Fig. 28, where $\lambda_{c}=\lambda_{s}$ is assumed. It is much easier to realize $C=1$ than in photonic crystals, since the mode expansion in the vertical direction is easily reduced to $\lambda / 2 n$ order by the strong optical confinement of the disk waveguide. For $\lambda_{s}=1.55 \mu \mathrm{m}$ and $\Delta \lambda=80 \mathrm{~nm}$, the condition is $D=1-2 \mu \mathrm{m}$. The cavity $Q$, which is affected by the diffraction loss was estimated using the approximate formula [90] with the equivalent index. As shown in Fig. 28, $Q$ is decreased rapidly as $D$ is further reduced from the above value. Spontaneous emission rate may be slightly increased for $D<\lambda$. But it will be saturated and decreased to less than 1 when the diameter is small so that the mode field expands to outer space and effective mode volume is increased.

\section{B. Fabrication and Evaluation of GaInAsP-InP Devices}

So far, photopumped lasing in a 1.6- $\mu$ m-diameter device has been reported and the $C$ factor of 0.2 has been estimated [89]. 


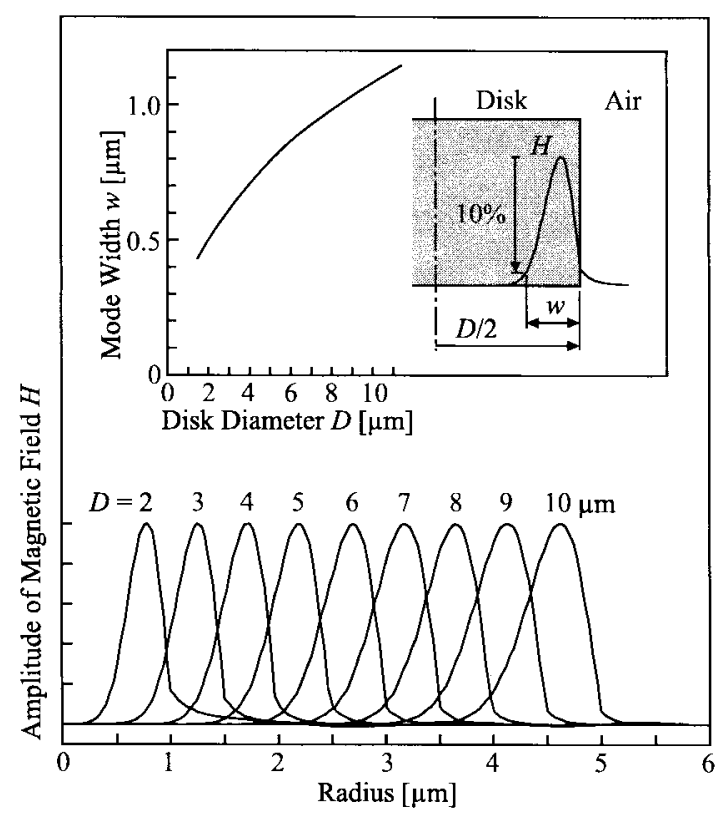

Fig. 29. Field distribution of whispering gallery mode and effective mode width $w$ for various disk diameters.

Fabrication of injection-type devices of 5-9 $\mu \mathrm{m}$ in diameter has also been reported [31], but the lasing operation was limited to those of no smaller than $9 \mu \mathrm{m}$. In the following, lasing operation of injection-type devices of $2-10 \mu \mathrm{m}$ is presented.

In the experiment, CS-MQW wafer described in Section IV was also used. For disk cavities, GRIN layers included in the active layer are expected to help the smooth carrier injection into CS-MQW and to suppress the recombination at disk surfaces. Between the substrate and n-InP cladding below the active layer, $0.3-\mu \mathrm{m}$-thick n-GaInAs was inserted to stop the wet etching described below. On p-InP cladding above the active layer, $1-\mu \mathrm{m}$-thick $\mathrm{p}-\mathrm{GaInAs}$ was grown as a contact layer. On the epitaxial surface $\mathrm{Au}-\mathrm{Zn}-\mathrm{Au}-\mathrm{Ti}$ was evaporated, while on the substrate surface AuGe. After patterning the Ti film into circular dot shapes, the $\mathrm{Au}-\mathrm{Zn}-\mathrm{Au}$ film was etched using Ar ion-beam etching and semiconductors to just above the n-GaInAs layer by $\mathrm{CH}_{4}$ based RIBE under the condition described in Section III. Formed circular mesas were nearly $4 \mu \mathrm{m}$ in height. After annealing the wafer for making ohmic contact, the disk shape was formed by the selective wet etching of InP claddings using $\mathrm{HCl}$ solution at $2{ }^{\circ} \mathrm{C}$. The disk width exposed to the air is $1 \mu \mathrm{m}$ for etching time approximated by $13 \mathrm{D}$ [s], where $D$ is in the unit of $\mu \mathrm{m}$. Fig. 29 shows simulated distributions of the highest order whispering gallery mode in the disk of $2-10 \mu \mathrm{m}$ in diameter. The effective mode width defined as shown in the inset is decreased by reducing $D$. To accelerate sufficient carrier diffusion into the disk edge, the disk width should be as small as possible within the extent that the mode does not suffer serious scattering loss at the InP pedestals. Disk width of all the fabricated devices was controlled to $0.5-1$ $\mu \mathrm{m}$. Fig. 30 shows the side view of fabricated devices. For larger devices, almost square cross section of InP pedestals caused by the anisotropic etching was observed. It may cause the scattering loss and inuniform carrier diffusion. For smaller devices, the cross section was almost circular. In general, it is easier to control the wet etching for smaller devices.
The diameter of the top contact of the $2-\mu \mathrm{m}$-diameter device was reduced to $1.2 \mu \mathrm{m}$ by the RIBE process. The current was flowed from a sharpened tungsten probe directly touching such a small top contact. The position of the probe was controlled by applying voltage between the probe and the top contact beforehand and monitoring the tunneling current. The radiation was directly detected by a sharpened single-mode fiber and analyzed by an optical spectrum analyzer. For smaller devices, the radiation was uniform inside the substrate plane. For devices larger than $5 \mu \mathrm{m}$ in diameter, it was uniform above threshold, but stronger in $\langle 110\rangle$ and $\langle 1 \overline{1} 0\rangle$ directions below threshold. The uniform radiation and the anisotropic radiation seem to be caused by the scattering of whispering gallery modes at disk edge and by the direct radiation from the square center region, respectively. In Fig. 31, open circles show the light output versus current characteristic of $2-\mu$ m-diameter device at 286 $\mathrm{K}$ under pulsed condition. The threshold current was estimated to $0.2 \mathrm{~mA}$. This value seems to be the lowest among those ever reported for GaInAsP lasers, even though considering the relatively low environmental temperature. However, the corresponding threshold current density is still as high as 6.4 $\mathrm{kA} / \mathrm{cm}^{2}$. Above threshold, the spectrum showed single-mode lasing with FWHM of $0.2 \mathrm{~nm}$, the resolution limit of the measurement. However, multimode lasing was often observed for diameter larger than $5 \mu \mathrm{m}$. For the $2-\mu \mathrm{m}$ device, the $C$ factor was calculated to be 0.4 , when assuming the polarization anisotropy of the CS-MQW wafer. In Fig. 31, theoretical curve with $C=0.4$ fits to experimental plots with small error. Excess power observed below threshold seems to be due to the radiation from the center region. For the precise evaluation of $C$ factor, a wide range evaluation of laser mode power below and above threhosld under continuous wave $(\mathrm{CW})$ condition and fitting to a theoretical curve are necessary. However, CW lasing was not obtained due to large heat caused by no heat sinking structure as well as large series resistance of $0.6-2 \mathrm{k} \Omega$.

The theoretical threshold current was calculated by taking the following nine properties of the disk laser into account.

1) Logarithmic Gain of $C S-M Q W$ : For current density $J$ and well number $N_{w}, G=G_{0} \ln \left(J / N_{w} J_{0}\right)$ with $G_{0}=$ $780 \mathrm{~cm}^{-1}$ and $J_{0}=74 \mathrm{~A} / \mathrm{cm}^{2}$ was assumed. It well explains the threshold current of stripe lasers fabricated in the same wafer.

2) Large optical confinement factor $\Gamma$ into $C S-M Q W$ : It was calculated for the disk waveguide to be $7.8 \%$. This value is 2.5 times larger than that for typical GaInAsP-InP waveguide.

3) Low absorption loss of nearly $5 \mathrm{~cm} \cdot \mathrm{cm}^{-1}$ owing to the air claddings of the disk waveguide.

4) Scattering Loss at Disk Surfaces and Edge: Scattering loss at disk surfaces may be much increased by large $\Delta n$ of the disk waveguide. Fig. 32 shows the scattering loss calculated from the perturbation theory [92], where normalized frequency $V=k_{c} n_{1}(d / 2) \sqrt{n_{1}^{2}-n_{2}^{2}}$ was fixed to 1.34 . It is estimated to be nearly $5 \mathrm{~cm}^{-1}$ when the surface roughness is $2 \mathrm{~nm}$. Similarly, $60 \mathrm{~cm}^{-1}$ was estimated for the edge roughness of $10 \mathrm{~nm}$.

5) Diffraction loss approximated in [90].

6) Recombination at p-side disk surface with velocity $v_{s}$. Electron concentration at p-side surface (GRIN layer surface) was assumed to be $20 \%$ of that in CS-MQW. 


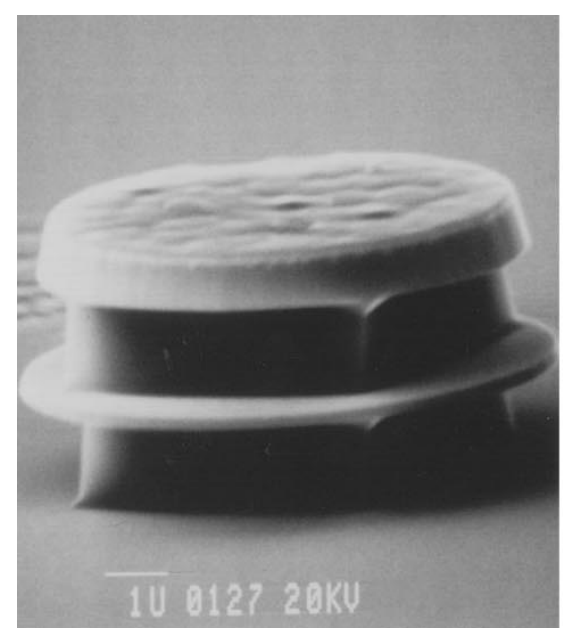

(a)

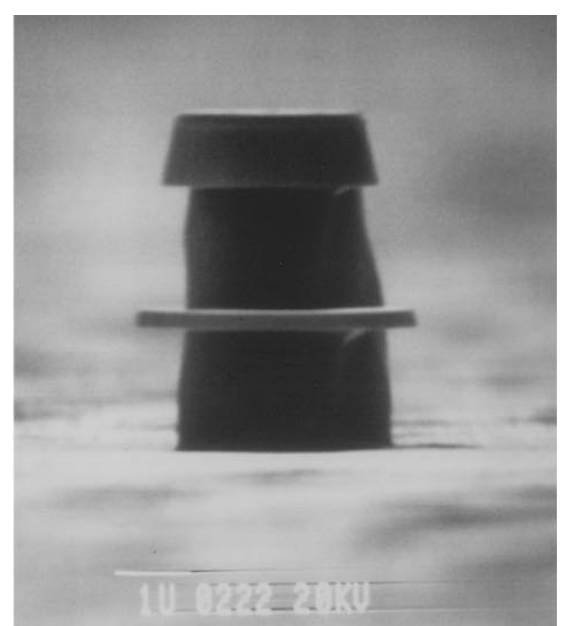

(b)

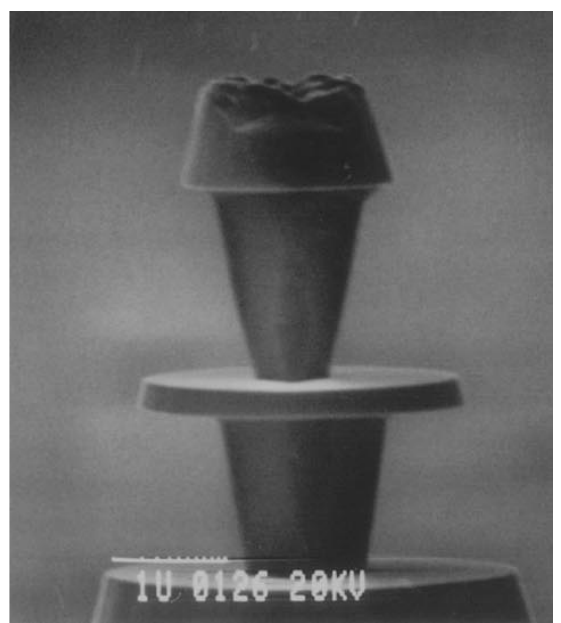

(c)

Fig. 30. SEM views of fabricated microdisk laser with diameter of (a) $8 \mu \mathrm{m}$, (b) $3 \mu \mathrm{m}$, and (c) $2 \mu \mathrm{m}$.

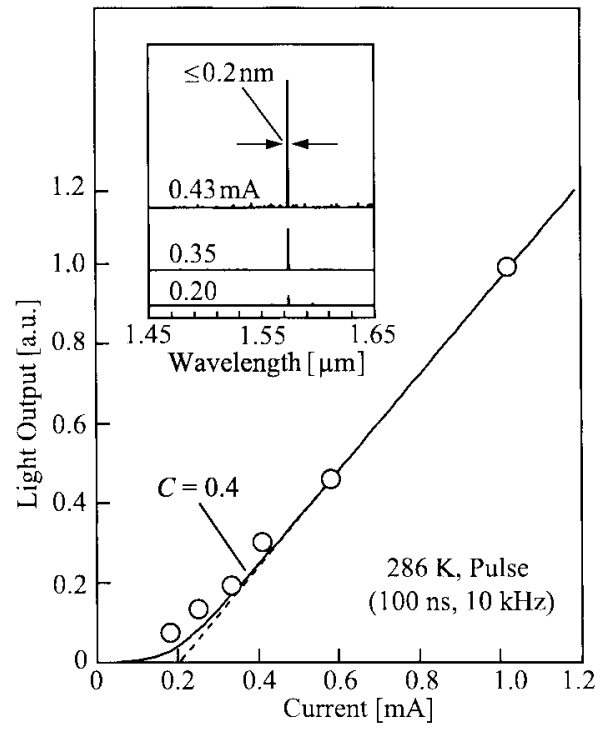

Fig. 31. Theoretical curve and experimental plots of light output versus current characteristic, and lasing spectra of $2-\mu \mathrm{m}$-diameter device.

7) Current passing through the center region, which does not contribute to laser oscillation.

8) Decay of carrier concentration toward the disk edge. It was approximated by $N_{\text {in }} \exp \left[-\left(r-r_{\text {in }}\right) / L_{\text {diff }}\right] r_{\text {in }} / r$, where $N_{\text {in }}$ and $r_{\text {in }}$ are carrier concentration and radius of the center region, respectively, and $L_{\text {diff }}$ is the diffusion length. In the calculation, $L$ was fixed to $2 \mu \mathrm{m}$.

9) Small spontaneous emission rate caused by the low effective index of the disk waveguide, as seen from (2). In the calculation, the effective index was assumed to be 2.65 .

Experimental threshold current was plotted with theoretical curves, as shown in Fig. 33. It was noticed in the calculation that threshold current is sensitive to the surface recombination velocity $v_{s}$ and the assumed carrier concentration at the GRIN layer surface as well as the total cavity loss. As seen in Fig. 33, experimental data fit to theoretical curves of $v_{s}=0.5-3 \times 10^{4}$ $\mathrm{cm} / \mathrm{s}$. Low threshold of $80 \mu \mathrm{A}$ is expected for diameter of 1.5 $\mu \mathrm{m}$ if the surface recombination is suppressed to negligible order by some passivation of disk surfaces [92].

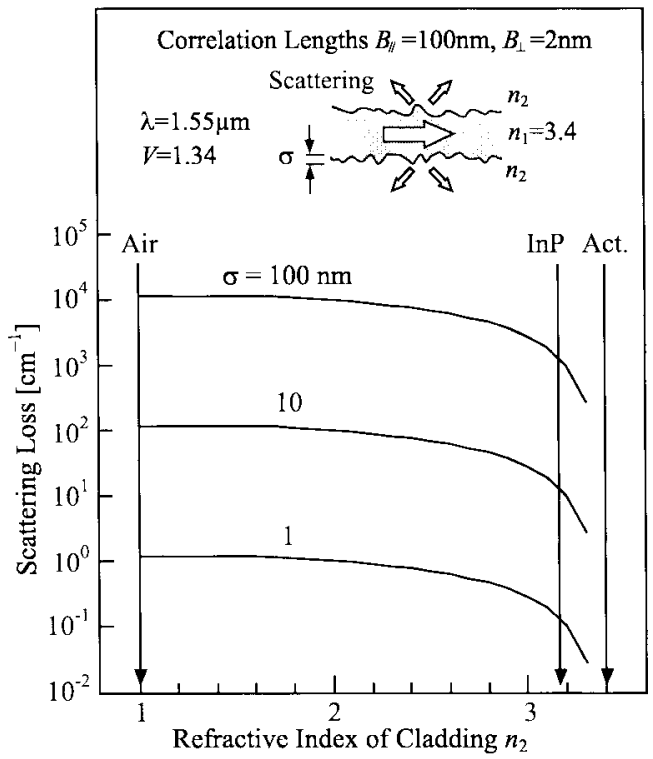

Fig. 32. Scattering loss of semiconductor/air waveguide with roughness amplitude of $\sigma$ and correlation length $B$.

Fig. 34 shows spontaneous emission spectra observed for $3-\mu$ m-diameter device under $\mathrm{cw}$ condition. Even when the current was as low as 20-50 $\mu \mathrm{A}$, sharp resonant peaks with FWHM of nearly $1 \mathrm{~nm}$ were observed. The cavity $Q$ calculated from this resonant width and the wavelength is 1595. This value almost accords with that estimated from the total cavity loss assumed in Fig. 33. For current of $200 \mu \mathrm{A}$, ratio of the main mode intensity to the background spontaneous emission was over $10 \mathrm{~dB}$. However, the FWHM was $0.5 \mathrm{~nm}$ and still wider than the resolution limit of the measurement. For this current, five resonant modes with wavelength separation of 27-38 $\mathrm{nm}$ were observed. When considering the nearly $4 \%$ free mode radiation toward upper and lower directions, this wavelength separation corresponds to $C$ factor of $0.33-0.46$ for spectral width $\Delta \lambda_{s}$ of $80 \mathrm{~nm}$, which was observed for GaInAsP-InP CS-MQW stripe lasers with injection current density nearly $1 \mathrm{kA} / \mathrm{cm}^{2}$. The wider $\Delta \lambda_{s}$ seen in Fig. 33 may be caused by the radiation from the center region with a higher carrier density than that at the disk edge. The arrows indicate 


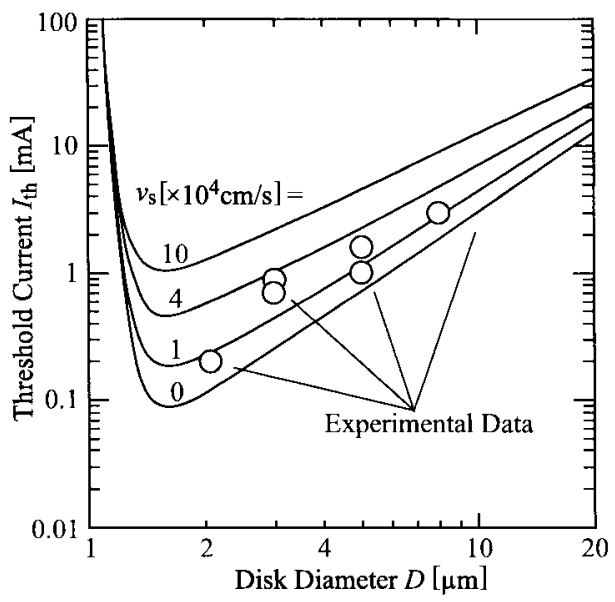

Fig. 33. Dependence of threshold current on disk diameter. Total cavity loss assumed is $70 \mathrm{~cm}^{-1}$.

resonant wavelengths predicted from the mode calculation. Accordance between the experiment and the theory was seen for three wavelengths, but rest two in the experiment shifted to longer wavelengths. This may be caused by the splitting of neighboring modes, which cannot be predicted by the approximate simulation. Two modes with narrow spectrum correspond to typical whispering gallery modes, as seen in Fig. 27(a) and (b), while the rest three with broad spectrum correspond to those rounding inside, as shown in Fig. 27(c) and (d). With current injection, the main mode blue-shifted at lower injection level and then red-shifted at higher injection level. These are caused by the plasma effect and the temperature increase, respectively. From the amount of the red shift, thermal resistance of this device is estimated to be 140000 $\mathrm{K} / \mathrm{W}$. The thermal resistance of GaInAsP-InP VCSEL's with diameter of $3 \mu \mathrm{m}$ was calculated to be $4000-20000 \mathrm{~K} / \mathrm{W}$ for various mirror materials [93], [94]. Even compared with the largest value, the evaluated thermal resistance for the disk laser is larger by factor 8 . It seems to be caused by the disk exposed to the air with no heat sinking structures, and will be improved by integrating a metal wire for the $p$-side contact.

In anyway, disk cavities have potentiality for thresholdless lasing operation by current injection even with present technologies. An interesting feature of this type of device is the independence of cavity loss from cavity size in the range that diffraction loss is negligible in compared to other losses, i.e., $D>0.8 \lambda(1.2 \mu \mathrm{m}$ for $\lambda=1.55 \mu \mathrm{m})$. This critical size will be realized without any special technical improvements but the reduction of sidewall inclination to obtain sufficient size of top contact. Precise theoretical estimation and experimental examination on cavity $Q$ and lasing performance under the ultimate condition $D<0.8 \lambda$ are the next issue.

\section{CONCLUSION}

The alternation of spontaneous emission rate is possible in a microcavity even with a broadening of electron transition spectrum, if the cavity size satisfies the single-mode condition that only one cavity mode is allowed in the transition spectrum. It originates from the enhancement of vacuum field amplitude in such an ultimate small cavity. Performance expected in a microcavity laser is summarized as follows:

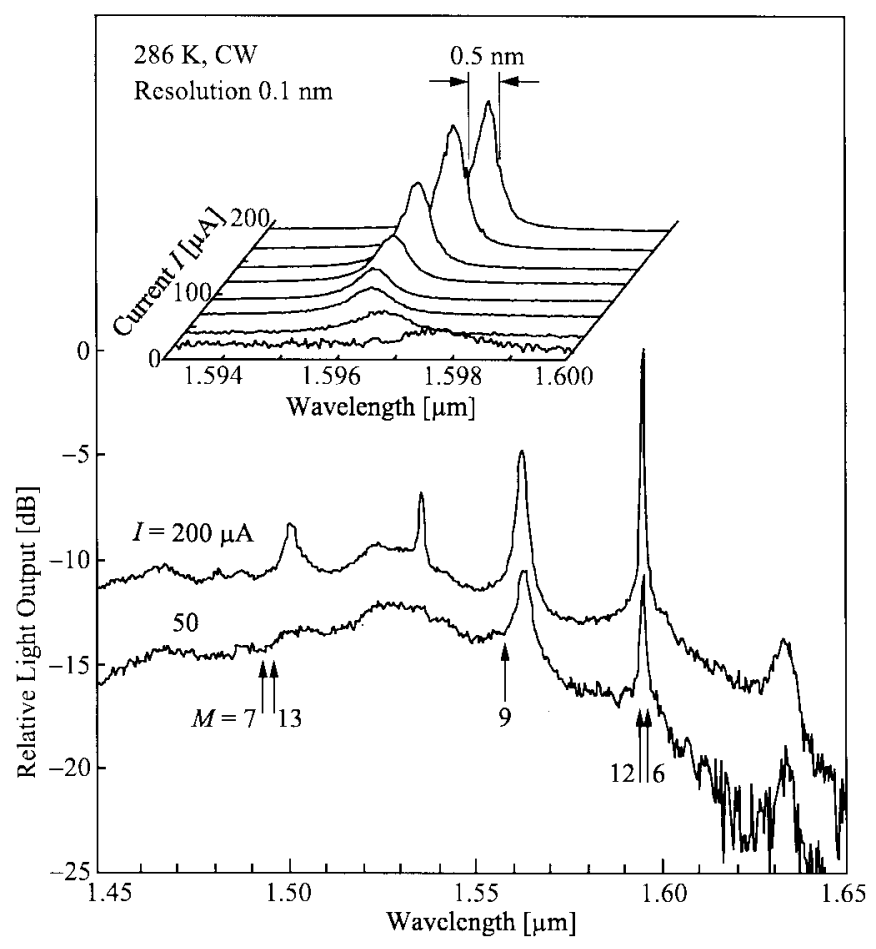

Fig. 34. Spontaneous emission spectra from $3-\mu$ m-diameter microdisk laser under $\mathrm{CW}$ condition.

1) thresholdless lasing operation; 2) LED-like operation with response comparable to those of laser diodes with relatively low pump density; and 3) ultrahigh-speed lasing operation with high pump density. Requirements for actual microcavities are the ultra-small mode volume, a cavity $Q$ at least satisfying $\Delta \omega_{l} \ll \Delta \omega_{s}$ and a a negligible surface recombination. For an ideal 3-D photonic crystal with one irregular atom, the thresholdless operation as well as the increase of spontaneous emission rate by factor 8 are expected. For 2-D crystals and whispering gallery mode cavities, the thresholdless operation is also expected, but the alternation of spontaneous emission rate may be negligible due to the insufficient optical confinement.

To obtain a high efficiency in microcavity laser for injection current lower than $1 \mathrm{~mA}$, surface recombination velocity less than $1 \times 10^{4} \mathrm{~cm} / \mathrm{s}$ is crucial. GaInAsP-InP system is expected to satisfy this condition. Fabrications of photonic crystals and whispering gallery mode cavities require a high aspect ratio over 10, smooth surfaces and/or sidewalls with roughness much less than $5 \mathrm{~nm}$, and adaptability to arbitrary shapes. At present, $\mathrm{CH}_{4}$-based RIBE seems to be the most promising method that exhibits sufficient performance for the latter two requirements and has room for the improvement of aspect ratio (presently 4) by a multilayer etching mask. A 2-D photonic crystal constructed by GaInAsP-InP columns was designed and fabricated using the RIBE. A polarized emission and peculiar transmission spectra were observed, which could be explained by the photonic band calculation. However, some additional effort is necessary to achieve perfect inhibition of spontaneous emission at photonic bandgaps and a high- $Q$ resonance applicable to lasers. In contrast to this, microdisk cavity, which is one of the whispering gallery mode cavities, easily achieves ultralow-threshold lasing operation with current injection, even when the size is very close to the 


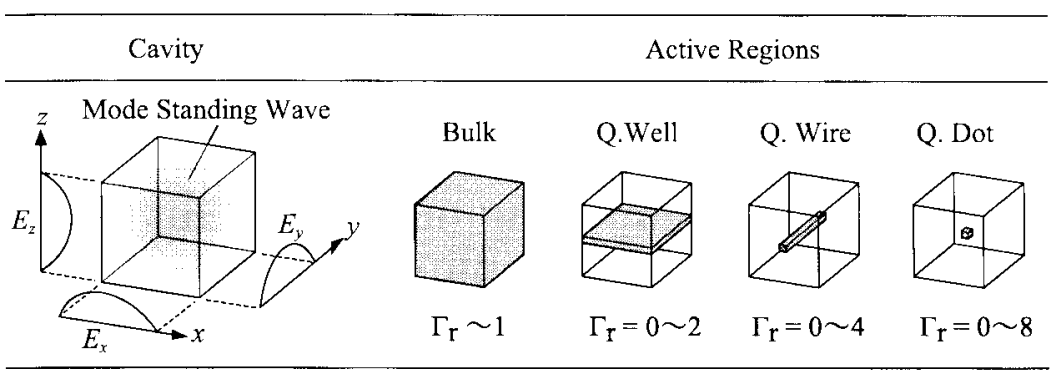

Fig. 35. Relation of relative confinement factor $\Gamma_{r}$, mode standing wave of cavity and active regions.

ultimate condition. In this study, threshold current of $0.2 \mathrm{~mA}$ was obtained for disk diameter of $2 \mu \mathrm{m}$. Sharp resonances were observed even with current less than $50 \mu \mathrm{A}$, which indicates the cavity $Q$ over 1500 . Precise theoretical estimation and experimental examination on cavity $Q$ and lasing performance under the ultimate condition are of great interest. For the experimental examination, $\mathrm{cw}$ lasing operation is crucial. Since there remains only some minor technical problems, they will soon be perfomed in future studies.

\section{APPENDIX A}

\section{STIMULATED AND SPONTANEOUS EMISSION RATES}

By some modification of Fermi's golden rule, the net recombination rate $R(\boldsymbol{r})$ of a dipole at position $\boldsymbol{r}$ is given by

$$
\begin{aligned}
R(\boldsymbol{r})= & \int_{0}^{\infty} \frac{2 \pi}{\hbar} e^{2} \mu^{2}(\boldsymbol{r}, \omega) E^{2}(\boldsymbol{r}, \omega)\left[\left(f_{2}-f_{1}\right)\right. \\
& \left.\cdot S V_{a} \delta\left(\omega-\omega_{l}\right)+f_{2}\left(1-f_{1}\right) \rho(\omega)\right] d \omega
\end{aligned}
$$

where $\mu$ is the amplitude of dipole moment, $E$ the electric field amplitude of light, $f_{2}$ and $f_{1}$ Fermi-Dirac distributions of conduction and valence bands, respectively, and $\rho$ the mode density. Assuming the uniform distribution of dipoles, the term $\mu^{2} f_{2}\left(1-f_{1}\right)$ is replaced by $\left(\mu_{0} / 3\right) F_{\mathrm{sp}}$, where $1 / 3$ is the probability of dipole orientation and $F_{\mathrm{sp}}$ is the transition spectrum normalized so that $\int_{0}^{\infty} F_{\mathrm{sp}} d \omega=1$. In a large cavity, $\rho$ is given as

$$
\rho d \omega=2 \frac{\omega^{2} V_{c} n_{\mathrm{eq}}^{2}}{2 \pi^{2} c^{2}} \cdot \frac{d k}{d \omega} d \omega=2 \frac{\omega^{2} V_{c} n_{\mathrm{eq}}^{2} n_{\mathrm{eff}}}{2 \pi^{2} c^{3}} \cdot d \omega
$$

where factor 2 indicates two polarizations. The equivalent index $n_{\mathrm{eq}}$ is given by

$$
\begin{aligned}
& 2 n_{\mathrm{eq}}^{2} \int_{\text {cavity }} \varepsilon_{0} E^{2}(\boldsymbol{r}, \omega) d^{3} \boldsymbol{r} \\
& \quad=2 \int_{\text {cavity }} \varepsilon_{0} n^{2}(\boldsymbol{r}) E^{2}(\boldsymbol{r}, \omega) d^{3} \boldsymbol{r}=\hbar \omega
\end{aligned}
$$

where $n(\boldsymbol{r})$ is the index distribution of cavity, and the right side is the normalization condition of electromagnetic energy of mode standing wave. $n_{\mathrm{eff}}$ is the effective index defined as $n_{\mathrm{eff}} \equiv n_{\mathrm{eq}}+\omega\left(d n_{\mathrm{eq}} / d \omega\right) . R_{\mathrm{sp}}$ is averaged by integrating the second term of (11) with respect to $\boldsymbol{r}$ in the active region and dividing by $V_{a}$. Using (12) and (13),

$$
R_{\mathrm{sp}}=\frac{e^{2} \mu_{0}^{2}}{3 \pi c^{3} \varepsilon_{0}} \int_{0}^{\infty} \omega^{3} n_{\mathrm{eff}} \Gamma_{r} F_{\mathrm{sp}} d \omega
$$

where $\Gamma_{r}$ is defined as [95]

$$
\Gamma_{r} \equiv \frac{1}{V_{a}} \int_{\text {active }} E^{2} d^{3} \boldsymbol{r} / \frac{1}{V_{c}} \int_{\text {cavity }} E^{2} d^{3} \boldsymbol{r}
$$

$\Gamma_{r}$ indicates how efficiently the mode standing wave overlaps with the active region. It is related to $\Gamma$ as

$$
\Gamma \equiv \int_{\text {active }} n_{a}^{2} E^{2} d^{3} \boldsymbol{r} / \int_{\text {cavity }} n^{2} E^{2} d^{3} \boldsymbol{r}=\frac{n_{a}^{2} V_{a}}{n_{\mathrm{eq}}^{2} V_{c}} \Gamma_{r}
$$

As shown in Fig. $35, \Gamma_{r}$ is maximally changed from $0-8$, depending on the relation of mode standing wave and the shape and position of active region. However, when the cavity has many modes, this change is averaged to 1 . For small broadening of $F_{\mathrm{sp}}$, (14) is simplified to

$$
R_{\mathrm{sp}}=\frac{e^{2} \mu_{0}^{2} \omega_{s}^{3} n_{\mathrm{eff}}}{3 \pi c^{3} \varepsilon_{0}}
$$

In a microcavity with discrete modes, the mode interval $\Delta \Omega$ is obtained by taking the reciprocal of (12) as

$$
\Delta \Omega=\frac{2 \pi^{2} c^{3}}{n_{\mathrm{eq}}^{3} V_{c} \omega^{2}}
$$

where factor 2 of (12) and the dispersion of $n_{\mathrm{eq}}$ were ignored. For cavities satisfying the semi-single mode condition of (3),

$$
R_{\mathrm{sp}}=\frac{2 \pi e^{2} \mu_{0}^{2} \omega_{c} \Gamma_{r}}{3 \varepsilon_{0} n_{\mathrm{eq}}^{2} V_{c}} \cdot \frac{\Delta \omega_{s} / 2}{\pi\left[\left(\omega_{s}-\omega_{c}\right)^{2}+\left(\Delta \omega_{s} / 2\right)^{2}\right]} .
$$

Taking average of the first term of (11) with respect to $r$ and using (13)

$$
R_{\mathrm{st}}=\frac{n_{a}^{2} V_{a}}{n_{\mathrm{eq}}^{2} V_{c}} \Gamma_{r} S \frac{\pi e^{2} \mu_{0}^{2} \omega_{l} F_{\mathrm{st}}}{3 n_{a}^{2} \varepsilon_{0}}=\Gamma S \frac{\pi c^{2} \mu_{0}^{2} \omega_{l} F_{\mathrm{st}}}{3 n_{a}^{2} \varepsilon_{0}} .
$$

\section{APPENDIX B}

\section{Spontaneous Emission Rate IN Photonic CRystals}

Solid angles $\Omega_{1}^{\text {leaky }}$ and $\Omega_{2}^{\text {leaky }}$ occupied by leaky mode radiations in 1-D and 2-D crystals, respectively, are given by

$$
\begin{aligned}
& \Omega_{1}^{\text {leaky }}=4 \pi\left(1-\Delta \lambda_{g} / 2 \lambda_{g}\right) \\
& \Omega_{2}^{\text {leaky }}=4 \pi\left[1-\sqrt{\left(\Delta \lambda_{g} / \lambda_{g}\right)-(1 / 4)\left(\Delta \lambda_{g} / \lambda_{g}\right)^{2}}\right] .
\end{aligned}
$$

The assumption for leaky modes in Section IV gives the ratio $\gamma$ in 1-D and 2-D crystals without irregular atoms as

$$
\gamma_{1,2}=\frac{n_{\mathrm{eff}}^{\text {leak }}}{n_{\mathrm{ref}}} \cdot \frac{\Omega_{1,2}^{\text {leak }}}{4 \pi}
$$




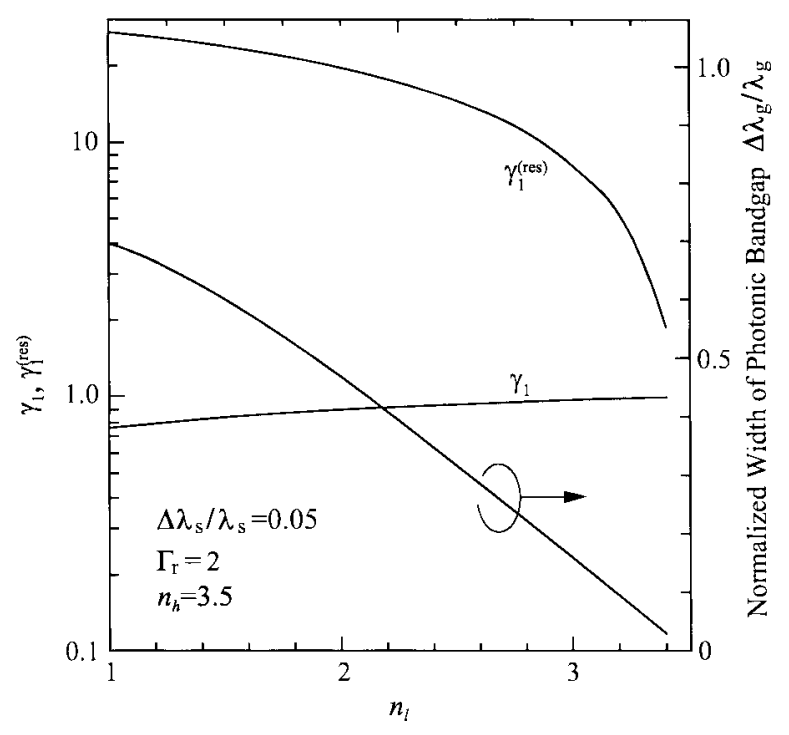

Fig. 36. Dependence of spontaneous emission rate and normalized width of photonic bandgap in 1-D crystal on refractive index of low index layer $n_{l}$.

where $n_{\text {eff }}^{\text {leak }}$ is the average effective index of leaky modes. For 3-D crystals,

$$
\gamma_{3}=\frac{n_{\mathrm{eff}}^{\text {leak }}}{n_{\mathrm{ref}}} \int_{\left|\lambda-\lambda_{g}\right|>\Delta \lambda_{g}} F_{\mathrm{sp}} d \lambda
$$

Resonant modes in 1-D crystals with an irregular atom have radiation solid angle of $2 \pi \Delta \lambda_{s} / \lambda_{s}=2 \pi \Delta \omega_{s} / \omega_{s} \cdot \gamma_{1}$ for all modes is derived as

$$
\gamma_{1}=\frac{n_{\mathrm{eff}}^{\text {leak }}}{n_{\mathrm{ref}}} \cdot \frac{\Omega_{1}^{\text {leak }}}{4 \pi}+\frac{n_{\mathrm{eq}}}{n_{\mathrm{ref}}} \cdot \frac{\Gamma_{r} \lambda_{s}}{4 n_{\mathrm{eq}} L_{z}}
$$

where $L_{z}$ is the effective mode length of resonant modes in the $z$ direction. Only for one resonant mode,

$$
\gamma_{1}^{\mathrm{res}}=\frac{n_{\mathrm{eq}}}{n_{\mathrm{ref}}} \cdot \frac{\Gamma_{r} \lambda_{s}^{2}}{2 n_{\mathrm{eq}} L_{z} \Delta \lambda_{s}} .
$$

From the theory of multilayer films [71],

$$
\frac{\Delta \lambda_{g}}{\lambda_{g}}=\frac{\Delta \omega_{g}}{\omega_{g}}=\frac{4}{\pi} \sin ^{-1}\left(\frac{n_{h}-n_{l}}{n_{h}+n_{l}}\right)
$$

where $n_{h}$ and $n_{l}$ are indexes of higher and lower index layers, respectively. Neglecting the dispersion of $n_{\mathrm{eq}}$,

$$
n_{\mathrm{eff}}^{\text {leak }} \sim \sqrt{n_{h} n_{l}}
$$

When $d_{c}$ is the multiple of $\lambda_{s} / n_{h}$ and $n_{\mathrm{eq}}=n_{h}, L_{z}$ is given by [96]

$$
L_{z}=d_{c}+\frac{\lambda_{s}}{2 n_{h}} \cdot \frac{n_{l}}{n_{h}-n_{l}} .
$$

Fig. 36 summarizes $\Delta \lambda_{g} / \lambda_{g}, \gamma_{1}^{\text {res }}$ and $\gamma_{1}$ calculated from (21)-(29). Smaller $n_{l}$ gives a large $\gamma_{1}^{\text {res }}$. Therefore, the emission intensity becomes stronger in the upper and lower directions, as demonstrated in Section III. However, $\gamma_{1}$ becomes rather small since changes of $\Omega_{1}^{\text {leak }}$ and $L_{z}$ cancel with each other and $n_{\mathrm{eff}}^{\text {leak }}$ is decreased.
In 2-D crystals, radiation solid angle of resonant mode is $4 \pi \sqrt{\Delta \lambda_{s} / \lambda_{s}}$, and

$$
\gamma_{2}=\frac{n_{\mathrm{eff}}^{\text {leaky }}}{n_{\mathrm{ref}}} \cdot \frac{\Omega_{2}^{\text {leaky }}}{4 \pi}+\frac{n_{\mathrm{eq}}}{n_{\mathrm{ref}}} \frac{\Gamma_{r} \lambda_{s}^{2}}{4 \pi n_{\mathrm{eq}}^{2} L_{x} L_{y} \sqrt{\Delta \lambda_{s} / \lambda_{s}}} .
$$

For one resonant mode,

$$
\gamma_{2}^{\text {res }}=\frac{n_{\mathrm{eq}}}{n_{\mathrm{ref}}} \cdot \frac{\Gamma_{r} \lambda_{s}^{3}}{4 \pi n_{\mathrm{eq}}^{2} L_{x} L_{y} \Delta \lambda_{s}} .
$$

In 3-D crystals, radiation solid angle of resonant mode is $4 \pi$. If the volume of localized field is regarded as the cavity volume $V_{c}$, (4)-(6) can be used. Rewriting (5) into the same form as (25) and (30):

$$
\begin{aligned}
\gamma_{3}^{\text {res }}= & \frac{n_{\mathrm{eff}}^{\text {leak }}}{n_{\mathrm{ref}}} \int_{\left|\lambda-\lambda_{g}\right|>\Delta \lambda_{g}} F_{\mathrm{sp}} d \lambda+\frac{n_{\mathrm{eq}}}{n_{\mathrm{ref}}} \\
& \cdot \frac{\Gamma_{r} \lambda_{s}^{3}}{2 \pi^{2} n_{\mathrm{eq}}^{3} L_{x} L_{y} L_{z}\left(\Delta \lambda_{s} / \lambda_{s}\right)} .
\end{aligned}
$$

It is noticed from (27)-(29) that $\Delta \lambda_{g} / \lambda_{g}$ for 1-D crystals is nearly equal to the mode interval $\lambda_{g} / 2 n_{\mathrm{eq}} L_{z}$ for Fabry-Perot cavity with mirror interval of $L_{z}$, when assuming $n_{\text {eff }}^{\text {leaky }} \sim n_{\mathrm{eq}}$ and $d_{c}=\lambda_{g} / n_{h}$. Let me consider the same correspondence to 2-D and 3-D. The mode interval for 3-D is given by (18), which can be rewritten as $\Delta \lambda_{g} / \lambda_{g} \sim \lambda_{g}^{3} / 4 \pi n_{\mathrm{eq}}^{3} L_{x} L_{y} L_{z}$. For 2-D, $\Delta \lambda_{g} / \lambda_{g} \sim \lambda_{g}^{2} / 2 \pi n_{\mathrm{eq}}^{2} L_{x} L_{y}$. Using (25), (30), and (32) and radiation solid angle of resonant mode for each structure, $\gamma$ and $\beta$ are obtained for $\Delta \lambda_{g} / \lambda_{g}=\Delta \omega_{g} / \omega_{g}$, as shown in Fig. 15.

\section{ACKNOWLEDGMENT}

The author would like to thank Prof. Y. Kokubun and Prof. Y. Hirose, Yokohama National University, and Prof. K. Iga, Prof. S. Arai, and Assoc. Prof. F. Koyama, Tokyo Institute of Technology, for encouragement and valuable discussions. He also thanks Mr. A. Matsutani, Tokyo Institute of Technology, and Dr. S. Kashiwa, Dr. T. Ninomiya, and Dr. A. Kasukawa, The Furukawa Electronic Company, Ltd., for help with the experiment.

\section{REFERENCES}

[1] E. M. Purcell, "Spontaneous emission probabilities at radio frequencies," Phys. Rev., vol. 69, p. 681, 1946.

[2] E. T. Jaynes and F. W. Comings, "Comparison of quantum and semiclassical radiation theories with application to the beam maser," Proc. IEEE, vol. 51, pp. 89-109, 1963.

[3] K. H. Drexhage, "Interaction of light with monomolecular dye layers,' Progress in Optics E. Wolf, Ed. New York: North-Holland, 1974, vol. 12, pp. $165-232$.

[4] P. Goy, J. M. Raimond, M. Gross, and S. Haroche, "Observation of cavity-enhanced single-atom spontaneous emission," Phys. Rev. Lett., vol. 50, pp. 1903-1909, 1983.

[5] R. G. Hulet, E. S. Hilfer, and D. Kleppner, "Inhibited spontaneous emission by Rydberg atom," Phys. Rev. Lett., vol. 55, pp. 2137-2140, 1985.

[6] T. Kobayashi, T. Segawa, Y. Morimoto, and T. Sueta, "Novel-type lasers, emitting devices, and functional optical devices by controlling spontaneous emission," in 46th Fall Meet. Jpn Soc. of Applied Physics, 1982, no. 29a-B-6 (in Japanese).

[7] E. Yablonovitch, "Inhibited spontaneous emission in solid-state physics and electronics," Phys. Rev. Lett., vol. 58, pp. 2059-2062, 1987.

[8] E. Yablonovitch, T. J. Gmitter, R. D. Meade, A. M. Rappe, K. D. Brommer, and J. D. Joannopoulos, "Donor and acceptor modes in photonic band structure," Phys. Rev. Lett., vol. 67, pp. 3380-3383, 1991. 
[9] S. John, "Strong localization of photons in certain disordered dielectric superlattices," Phys. Rev. Lett., vol. 58, pp. 2486-2489, 1987.

[10] F. De Martini, G. Innocenti, G. R. Jacobovitz, and P. Mataloni, "Anomalous spontaneous emission time in a microscopic optical cavity," Phys. Rev. Lett., vol. 59, pp. 2955-2958, 1987.

[11] F. De Martini and G. R. Jacobovitz, "Anomalous spontaneousstimulated-decay phase transition and zero-threshold laser action in a microscopic cavity," Phys. Rev. Lett., vol. 60, pp. 1711-1714, 1988.

[12] K. Iga, F. Koyama, and S. Kinoshita, "Surface emitting semiconductor lasers," IEEE J. Quantum Electron., vol. 24, pp. 1845-1855, 1988.

[13] F. Koyama, S. Kinoshita, and K. Iga, "Room-temperature CW operation of GaAs vertical cavity surface emitting laser," Trans. IEICE., vol. E7, pp. 1089-1090, 1988.

[14] J. L. Jewell, A. Scherer, S. L. McCall, Y. H. Lee, S. J. Walker, J. P. Harbison, and L. T. Florez, "Low-threshold electrically pumped vertical-cavity surface emitting microlasers," Electron. Lett., vol. 25, pp. 1123-1124, 1989.

[15] Y. Yamamoto, S. Machida, K. Igeta, and Y. Horikoshi, "Enhanced and inhibited spontaneous emission of free excitons in GaAs quantum wells in a micro-cavity," Proc. 6th Rochester Conf. Quantum Opt., 1987.

[16] Y. Yamamoto, S. Machida, K. Igeta, and G. Björk, "Controlled spontaneous emission in microcavity semiconductor lasers," in Coherence, Amprification and quantum effects in semiconductor lasers Y. Yamamoto, Ed. New York: Wiley, 1991

[17] H. Yokoyama and S. D. Brorson, "Rate equation analysis of microcavity lasers," J. Appl. Phys., vol. 66, pp. 4801-4805, 1989.

[18] G. Björk and Y. Yamamoto, "Analysis of semiconductor microcavity lasers using rate equations," IEEE J. Quantum Electron., vol. 27, pp. 2386-2396, 1991

[19] M. Yamanishi, Y. Yamamoto, and T. Shiotani, "A novel modulation scheme in semiconductor light emitters with quantum microcavities: High speed intensity modulation by switching of coupling efficiency of spontaneous emission," IEEE Photon. Technol. Lett., vol. 3, pp. 888-890, 1991

[20] G. P. Agrawal and G. R. Gray, "Intensity and phase noise in microcavity surface-emitting semiconductor laser," Appl. Phys. Lett., vol. 59, pp 399-401, 1991.

[21] X. P. Feng and K. Ujihara, "Quantum theory of spontaneous emission in a one-dimensional optical cavity with two-side output coupling," Phys. Rev. A, vol. 41, pp. 266-2676, 1990.

[22] T. Baba, T. Hamano, F. Koyama, and K. Iga, "Spontaneous emission factor of a microcavity DBR surface emitting laser," IEEE J. Quantum Electron., vol. 27, pp. 1347-1358, 1991.

[23] _ "Spontaneous emission factor of a microcavity DBR surface emitting laser (II)-Effect of electron quantum confinements," IEEE J. Quantum Electron., vol. 28, pp. 1310-1319, 1992.

[24] T. Numai, K. Kurihara, I. Ogura, H. Kosaka, M. Sugimoto, and K. Kasahara, "Effect of sidewall reflector on current versus light output in a pnpn vertical-to-surface transmission electrophotonic device with a vertical cavity," IEEE J. Quantum Electron., vol. 29, pp. 2006-2012, 1993.

[25] T. Tamanuki, F. Koyama, and K. Iga, "Threshold and modulation characteristics of photon-recycled semiconductor lasers," Jpn. J. Appl. Phys., vol. 31, pp. 1810-1811, 1992.

[26] T. Baba, R. Watanabe, K. Asano, F. Koyama, and K. Iga, "Theoretical and experimental estimations of pho-ton recycling effect in light emitting devices with a metal mirror," Jpn. J. Appl. Phys., vol. 35, pp. 97-100, 1996.

[27] H. Yokoyama, K. Nishi, T. Anan, H. Yamada, S. D. Brorson, and E. P. Ippen, "Enhanced spontaneous emission from GaAs quantum wells with monolithic optical microcavities," Appl. Phys. Lett., vol. 57, pp. 2814-2816, 1990

[28] T. J. Rogers, D. G. Deppe and B. G. Streetman, "Effect of an AlAs/GaAs mirror on the spontaneous emission of an InGaAs-GaAs quantum well," Appl. Phys. Lett., vol. 57, pp. 1858-1860, 1990.

[29] C. Weisbuch, M. Nishioka, A. Ishikawa, and Y. Arakawa, "Observation of the coupled exciton-photon mode splitting in a semiconductor quantum microcavity," Phys. Rev. Lett., vol. 69, pp. 3314-3317, 1992.

[30] A. Scherer, J.L. Jewell, and J.P. Harbison, "Lasing in sub-micron wide vertical cavity microlasers," Opt. Photon. News, vol. 2, p. 9, 1991.

[31] A. F. J. Levi, R. E. Slusher, S. L. McCall, T. Tanbun-Ek, D. L. Coblentz, and S. J. Pearton, "Room temperature operation of microdisk lasers with submilliamp threshold current," Electron. Lett., vol. 28, pp. 1010-1012, 1992.

[32] A. F. J. Levi, R. E. Slusher, S. L. McCall, S. J. Pearton, and W. S. Hobson, "Room temperature lasing in $\mathrm{In}_{0.51} \mathrm{Ga}_{0.49} \mathrm{P} / \mathrm{In}_{0.2} \mathrm{Ga}_{0.8} \mathrm{As}$ microcylinder laser diodes," Appl. Phys. Lett., vol. 62, pp. 2021-2023, 1993.
[33] P. L. Gourley, J. R. Wendt, G. A. Vawter, T. M. Brennan, and B. E. Hammons, "Optical properties of two-dimensional photonic lattices fabricated as honeycomb nanostructures in compound semiconductors," Appl. Phys. Lett., vol. 64, pp. 687-689, 1994.

[34] T. Krauss, Y. P. Song, S. Thoms, C. D. W. Wilkinson, and R. M. DelaRue, "Fabrication of 2-D photonic bandgap structures in GaAs/AlGaAs," Electron. Lett., vol. 30, pp. 1444-1446, 1994.

[35] T. Baba and T. Matsuzaki, "Polarization change in spontaneous emission from GaInAsP/InP 2-dimensional photonic crystals," Electron. Lett., vol. 31, pp. 1776-1778, 1995.

[36] C. C. Cheng, A. Scherer, V. A. Engels and E. Yablonovitch, "Lithographic band gap tuning in photonic band gap crystals," J. Vac. Sci. Technol. B, vol. 14, pp. 4110-4114, 1996.

[37] H. Yokoyama and K. Ujihara, Eds., Spontaneous Emission and Laser Oscillation in Microcavities. New York: CRC, 1995.

[38] K. Asakawa and S. Sugata, "GaAs and AlGaAs anisotropic fine pattern etching using a new reactive ion beam etching system," J. Vac. Sci. Technol. B, vol. 3, pp. 402-405, 1988.

[39] A. Scherer, J. L. Jewell, Y. H. Lee, J. P. Harbison, and L. T. Florez, "Fabrication of microlasers and microresonator optical switches," Appl. Phys. Lett., vol. 55, pp. 2724-2726, 1989.

[40] E. Yablonovitch, T. J. Gmitter and R. Bhat, "Inhibited and enhanced spontaneous emission from optically thin $\mathrm{AlGaAs} / \mathrm{GaAs}$ double heterostructures," Phys. Rev. Lett., vol. 61, pp. 2546-2549, 1988

[41] Y. Suematsu and K. Furuya, "Theoretical spontaneous emission factor of injection lasers," Trans. IEICE Jpn., vol. 60, pp. 467-472, 1977.

[42] F. Stern and J. M. Woodall, "Photon recycling in semiconductor lasers," J. Appl. Phys., vol. 45, pp. 3904-3906, 1974.

[43] T. Baba, Y. Yogo, K. Suzuki, F. Koyama, and K. Iga, "Near room temperature continuous wave lasing characteristics of GaInAsP/InP surface emitting laser," Electron. Lett., vol. 29, pp. 913-914, 1993.

[44] S. Uchiyama and S. Kashiwa, "GaInAsP/InP square buriedheterostructure surface-emitting lasers regrown by MOCVD," IEICE Trans. Electron., vol. E78C, pp. 1311-1313, 1995.

[45] S. Uchiyama, N. Yokouchi and T. Ninomiya, "Continuous-wave operation up to $36^{\circ} \mathrm{C}$ of $1.3-\mu \mathrm{m}$ GaInAsP-InP vertical-cavity surface-emitting lasers," IEEE Photon. Technol. Lett., vol. 9, pp. 141-142, 1997.

[46] J. J. Dudley, M. Ishikawa, D. I. Babic, B. I. Miller, R. Mirin, W. B. Jiang, J. E. Bowers, and E. L. Hu, " $144^{\circ} \mathrm{C}$ operation of $1.3 \mu \mathrm{m}$ InGaAsP vertical cavity lasers on GaAs substrates," Appl. Phys. Lett., vol. 61, pp. 3095-3097, 1992.

[47] N. M. Margalit, D. I. Babic, K. Streubel, R. P. Mirin, R. L. Naone, J E. Bowers, and E. L. Hu, "Submilliamp long wavelength vertical cavity lasers," Electron. Lett., vol. 32, pp. 1675-1676, 1996.

[48] K. Streubel, S. Rapp, J. André, and N. Chitica, "1.26 $\mu \mathrm{m}$ vertical cavity laser with two InP/air-gap reflectors," Electron. Lett., vol. 32, pp. 1369-1370, 1996.

[49] T. Baba, M. Hamasaki, N. Watanabe, P. Kaewplung, A. Matsutani, T. Mukaihara, F. Koyama, and K. Iga, "A novel short cavity laser with deep grating distributed Bragg reflectors," Jpn. J. Appl. Phys., vol. 35, pp. 1390-1394, 1996.

[50] J. O'Brien, O. Painter, R. Lee, C. C. Cheng, A. Yariv, and A. Scherer, "Lasers incorporating 2D photonic bandgap mirrors," Electron. Lett., vol. 32, pp. 2243-2244, 1997.

[51] T. Baba and M. Koma, "Possibility of InP-based 2-dimensional photonic crystal-An approach by anodization technique," Jpn. J. Appl. Phys., vol. 34, pp. 1405-1408, 1995.

[52] T. Baba, N. Kamizawa, and M. Ikeda, "Nanofabrication process of GaInAsP/InP 2D photonic crystals by a methane-based reactive ion beam etching technique," Physica B, vol. 227, pp. 415-418, 1996.

[53] T. Hamano, H. Hirayama, and Y. Aoyagi, "Observation of photonic bandgap in GaAs 2D crystal fabricated by selective growth," in Proc. Topical Meet. Quantum Optoelectronics, 1997, vol. 9, pp. 29-31.

[54] S. Noda, N. Yamamoto, and A. Sasaki, "New realization method for three-dimensional photonic crystal in optical wavelength region," Jpn. J. Appl. Phys., vol. 35, pp. L909-L912, 1996.

[55] S. Mitsugi, F. Koyama J. Kato, A. Matsutani, T. Mukaihara, and K. Iga, "GaInAs/GaAs micro-arc ring semiconductor laser," Jpn. J. Appl. Phys., vol. 34, pp. 1265-1269, 1995.

[56] S. Ando, N. Kobayashi, and H. Ando, "Novel hexagonal-facet GaAs/AlGaAs laser grown by selective area metalorganic chemical vapor deposition," Jpn. J. App. Phys., vol. 32, pp. L1293-L1296, 1993.

[57] J. P. Zhang, D. Y. Chu, S. L. Wu, S. T. Ho, W. G. Bi, C. W. Tu, and R. C. Tiberio, "Photonic-wire laser," Phys. Rev. Lett., vol. 75, pp. 2678-2681, 1995.

[58] T. Baba, M. Fujita, A. Sakai, M. Kihara, and R. Watanabe, "Lasing characteristics of GaInAsP/InP strained quantum-well microdisk injec- 
tion lasers with diameter of 2-10 $\mu \mathrm{m}, "$ IEEE Photon. Technol. Lett., vol. 9, pp. 878-880, 1997.

[59] T. Tamanuki, F. Koyama, and K. Iga, "Interface recombination reduction by $\left(\mathrm{NH}_{4}\right)_{2} \mathrm{~S}_{x}$-passivation in metalorganic chemical vapor deposition regrown GaAlAs/GaAs buried heterostructure lasers and estimation of threshold currents in microcavity surface emitting lasers," Jpn. J. Appl. Phys., vol. 31, pp. 3292-3295, 1992.

[60] T. Tezuka, S. Nunoue, H. Yoshida, and T. Noda, "Spontaneous emission enhancement in pillar-type microcavities," Jpn. J. Appl. Phys., vol. 32, pp. L54-L57, 1993.

[61] B. E. Maile, A. Forchel and R. Germann, "Impact of sidewall recombination on the quantum efficiency of dry etched InGaAs/InP semiconductor wires," Appl. Phys. Lett., vol. 54, pp. 1552-1554, 1989.

[62] L. A. Coldren and J. A. Rentschler, "Directional reactive-ion-etching of InP with $\mathrm{Cl}_{2}$ containing gases," J. Vac. Sci. Technol., vol. 19, pp. 225-229, 1981.

[63] T. Tadokoro, F. Koyama, and K. Iga, "Classification of etching mechanism in reactive ion beam etch," J. Vac. Sci. Technol. B, vol. 7, pp. 1111-1114, 1989.

[64] A. Matsutani, F. Koyama, and K. Iga, "Characterization of sidewall damage induced by reactive ion-beam etching," Jpn. J. Appl. Phys., vol. 31, pp. 1541-1544, 1992.

[65] C. Constantine, D. Johnson, S. J. Pearton, U. K. Chakrabarti, A. B. Emerson, W. S. Hobson, and A. P. Kinsella, "Plasma etching of III-V semiconductors in $\mathrm{CH}_{4} / \mathrm{H}_{2} /$ Ar electron cyclotron resonance discharges," J. Vac. Sci. Technol. B, vol. 8, pp. 596-606, 1990.

[66] S. J. Pearton, U. K. Chakrabarti, A. Katz, A. P. Perley, W. S. Hobson, and C. Constantine, "Comparison of $\mathrm{CH}_{4} / \mathrm{H}_{2} / \mathrm{Ar}$ reactive ion etching and electron cyclotron resonance plasma etching of In-base III-V alloys," J. Vac. Sci. Technol. B, vol. 9, pp. 1421-1432, 1991.

[67] L. A. Coldren, K. Furuya, B. I. Miller, and J. A. Rentschler, "Combined dry and wet etching techniquess to form planar (011) facets in GaInAsP/InP double heterostructures," Electron. Lett., vol. 18, pp. 235-237, 1982

[68] K. Furuya, L. A. Coldren, B. I. Miller, and J. A. Rentschler, "Crystallographic facets chemically etched in GaInAsP/InP for integrated optics," Electron. Lett., vol. 17, pp. 582-583, 1981.

[69] K. Iga and B. I. Miller, "Chemically etched-mirror GaInAsP/InP lasers-Review," IEEE J. Quantum Electron., vol. QE-18, pp. 22-29, 1982

[70] T. Takizawa, S. Arai, and M. Nakahara, "Fabrication of vertical and uniform-size porous InP structure by electrochemical anodization," Jpn. J. Appl. Phys., vol. 33, L643-L645, 1994.

[71] Z. Knittl, "Optics of thin films," An Optical Multilayer Theory. New York: Wiley-Interscience, 1976.

[72] A. Matsutani, F. Koyama, and K. Iga, "Three-dimensional electron probe roughness analysis of InP sidewalls processed by reactive ion beam etching," Appl. Phys. Lett., vol. 66, pp. 64-66, 1995.

[73] C. K. Carniglia, "Scalar scattering theory for multilayer optical coatings," Opt. Eng., vol. 18, pp. 104-114, 1979.

[74] M. Cantagrel, "Comparison of the properties of different materials used as masks for ion-beam etching," J. Vac. Sci. Technol., vol. 12, pp. 1340-1343, 1975.

[75] J. D. Joannopoulos, R. D. Meade, and J. N. Winn, Photonic Crystals. Princeton, NJ: Princeton Univ. Press, 1995.

[76] C. M. Bowden and J. P. Dowling, Eds., "Development and applications of materials exhibiting photonic band gaps," J. Opt. Soc. Amer. B, vol. 10-2, pp. 283-413, 1993.

[77] C. M. Soukoulis, Ed., Photonic Band Gap Materials. Dordrecht, The Netherlands: Kluwer Academic, 1996.

[78] J. Rarity and C. Weisbuch, Eds.,Microcavities and Photonic Bandgaps: Physics and Applications. Dordrecht, The Netherlands: Kluwer Academic, 1996.

[79] R. Biswas, C. T. Chan, M. Sigalas, C. M. Soukoulis, and K. M. Ho, "Photonic band gap materials," Photonic Band Gap Materials, C. M. Soukoulis, Ed. Dordrecht, The Netherlands: Kluwer Academic, 1996, pp. $23-40$.

[80] K. M. Leung and Y. F. Liu, "Full vector wave calculation of photonic band structures in face-centered-cubic dielectric media," Phys. Rev. Lett., vol. 65, pp. 2646-2649, 1990.

[81] Z. Zhang and S. Satpathy, "Electromagnetic wave propagation in periodic structures: Bloch wave solution of Maxwell's equations," Phys. Rev. Lett., vol. 65, pp. 2650-2653, 1990.

[82] K. M. Ho, C. T. Chan, and C. M. Soukoulis, "Existence of a photonic gap in periodic dielectric structures," Phys. Rev. Lett., vol. 65, pp. 3152-3155, 1990.
[83] P. R. Villeneuve and M. Piché, "Photonic bandgaps in periodic dielectric structures," Prog. Quantum Electron., vol. 18, pp. 153-200, 1994.

[84] M. Plihal and A. A. Maradudin, "Photonic band structure of twodimensional system: the triangular lattice," Phys. Rev. B, vol. 44, pp. 8565-8571, 1991.

[85] R. D. Meade, K. D. Brommer, A. M. Rappe, and J. D. Joannopoulos, "Existence of a photonic band gap in two dimensions," Appl. Phys. Lett., vol. 61, pp. 495-497, 1992.

[86] B. E. Maile, A. Forchel, R. Germann, and D. Grützmacher, "Impact of sidewall recombination on the quantum efficiency of dry etched InGaAs/InP semiconductor wires," Appl. Phys. Lett., vol. 54, pp. 1552-1554, 1989.

[87] E. P. ÓReilly and A. R. Adams, "Band-structure engineering in strained semiconductor lasers," IEEE J. Quantum Electron., vol. 30, pp. 366-379, 1994.

[88] T. Baba and T. Matsuzaki, "Fabrication and photoluminescence studies of GaInAsP/InP 2-dimensional pho-tonic crystals," Jpn. J. Appl. Phys., vol. 35, pp. 1348-1352, 1996.

[89] A. F. J. Levi, S. L. McCall, S. J. Pearton, and R. A. Logan, "Room temperature operation of submicrometer radius disk laser," Electron. Lett., vol. 29, pp. 1666-1667, 1993.

[90] S. L. McCall, A. F. J. Levi, R. E. Slusher, S. J. Pearton, and R. A. Logan, "Whispering gallery mode microdisk lasers," Appl. Phys. Lett., vol. 60, pp. 289-291, 1992.

[91] Y. Suematsu and K. Furuya: "Propagation mode and scattering loss of a two-dimensional dielectric waveguide with gradual distribution of refractive index," IEEE Trans. Microwave Theory Tech., vol. MTT-20, pp. 524-531, 1972.

[92] U. Mohideen, W. S. Hobson, S. J. Pearton, F. Ren, and R. E. Slusher, "GaAs/AlGaAs microdisk lasers," Appl. Phys. Lett., vol. 64, pp. 1911-1913, 1994.

[93] T. Baba, T. Kondo, F. Koyama, and K. Iga, "Finite element analysis of thermal characteristics in continuous wave long wavelength surface emitting lasers (I)—Dielectric cavity structures," Opt. Rev., vol. 2, pp. 123-127, 1995.

[94] T. Baba, T. Kondo, F. Koyama, and K. Iga, "Finite element analysis of thermal characteristics in continuous wave long wavelength surface emitting lasers (II)-Semiconductor distributed Bragg reflectors," Opt. Rev., vol. 2, pp. 323-325, 1995.

[95] S. W. Corzine, R. S. Geels, J. W. Scott, R. H. Yan, and L. A. Coldren, "Design of Fabry-Perot surface-emitting lasers with a periodic Gain Structure," IEEE J. Quantum Electron., vol. 25, pp. 1513-1524, 1989.

[96] D. I. Babic and S. T. Corzine, "Analytical expressions for the reflection delay, penetration depth, and absorptance of quarter-wave dielectric mirrors," IEEE J. Quantum Electron., vol. 28, pp. 514-524, 1992.

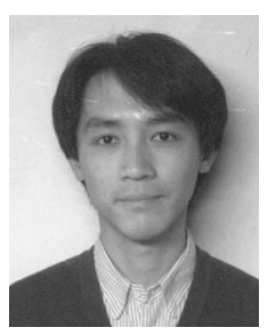

Toshihiko Baba (M'93) was born in Nagano Prefecture, Japan, on November 12, 1962. He received the B.E., M.E., and Ph.D. degrees from the Division of Electrical and Computer Engineering, Yokohama National University, Japan, in 1985 1987, and 1990, respectively.

During Ph.D. work, he had been engaged in antiresonant reflecting optical waveguides (ARROW's) and integrated lightwave circuits, From 1990, has joined the Precision and Intelligence Laboratory, Tokyo Institute of Technology, as a Research Associate, and started the research on VCSEL's. In 1991, he attracted attention on the first calculation of spontaneous emission factor in VCSEL's. In 1993, he achieved the first RT CW operation of long wavelength VCSEL. Now, he is an Associate Professor at Yokohama National University and also a Guest Associate Professor at the Tokyo Institute of Technology. His current interests are microcavity lasers, photonic band crystals, spontaneous emission control, micromechnical optics, and optical computing.

Dr. Baba is a member of the Institute of Electronics, Information and Communication Engineers (IEICE) and the Japan Society of Applied Physics. He received the Niwa Memorial Prize in 1991, the Best Paper Award of Micoropitic Conference in 1993, and the Paper Award and Academic Encouragement Award from the IEICE in 1994. 